\title{
Sur os et sur dents : les « retouchoirs » aurignaciens de la Ferrassie (Savignac-de- Miremont, Dordogne)
}

The utilization of bones and teeth as "retouchers " during the Aurignacian at La Ferrassie (Savignac-de-Miremont, Dordogne)

Jean-Christophe Castel, François-Xavier Chauvière et Stéphane Madelaine

OpenEdition

Journals

Édition électronique

URL : http://journals.openedition.org/paleo/1212

DOI : $10.4000 /$ paleo. 1212

ISSN : 2101-0420

Éditeur

SAMRA

Édition imprimée

Date de publication : 1 décembre 2003

Pagination : $29-50$

ISSN : 1145-3370

Référence électronique

Jean-Christophe Castel, François-Xavier Chauvière et Stéphane Madelaine, «Sur os et sur dents : les « retouchoirs » aurignaciens de la Ferrassie (Savignac-de-Miremont, Dordogne) », PALEO [En ligne], 15 | 2003, mis en ligne le 02 août 2010, consulté le 07 juillet 2020. URL : http://journals.openedition.org/ paleo/1212 ; DOI : https://doi.org/10.4000/paleo.1212

Ce document a été généré automatiquement le 7 juillet 2020

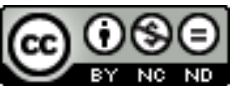

PALEO est mis à disposition selon les termes de la licence Creative Commons Attribution - Pas d'Utilisation Commerciale - Pas de Modification 4.0 International. 


\section{Sur os et sur dents : les " retouchoirs » aurignaciens de la Ferrassie (Savignac-de-Miremont, Dordogne)}

The utilization of bones and teeth as "retouchers » during the Aurignacian at La Ferrassie (Savignac-de-Miremont, Dordogne)

Jean-Christophe Castel, François-Xavier Chauvière et Stéphane Madelaine

\section{Introduction}

1 La mise en place des nouvelles salles d'exposition permanente du Musée national de Préhistoire des Eyzies a donné l'opportunité à l'un d'entre nous (J.-C.C.) d'effectuer un nouvel examen des collections fauniques du gisement de La Ferrassie (Savignac-deMiremont, Dordogne, fouilles Peyrony), en vue de leur présentation muséographique. A cette occasion, la re-découverte d'une canine de grand carnivore utilisée comme « cousoir » et publiée comme telle par L. Capitan et D. Peyrony (1912, p. 96, fig. 34 ; Peyrony 1934, p.51, fig. 49), s'est accompagnée de la mise au jour de trois dents semblables dans le niveau aurignacien à sagaies à base fendue (Aurignacien I ou Aurignacien ancien d'Aquitaine; $c f$. par exemple: Bon 2002; de Sonneville-Bordes 1960 ; Peyrony, 1933). Ces éléments sont associés à des dents jugales de cheval et à des diaphyses osseuses également porteuses de stigmates décrits, dans la littérature, comme caractéristiques des retouchoirs (Patou-Mathis 2002).

2 A la suite de son travail sur l'industrie osseuse aurignacienne, Christiane Leroy-Prost a publié récemment deux autres canines d'Ours de La Ferrassie, également issues des niveaux aurignaciens du gisement et conservées au Musée des Antiquités nationales de Saint-Germain-en-Laye, dans la collection Capitan (Leroy-Prost 1996, 2002). 
3 Au total, et jusqu'à ce jour, La Ferrassie a donc livré six canines de grands fauves (Ours et Lion), soit presque l'équivalent numérique du gisement de Vogelherd en Allemagne (Hahn 1977 ; Leroy-Prost 1996).

\section{1- Matériel d'étude}

\section{1- La Ferrassie : le site et la faune}

Figure 1 - Carte des sites ayant livré des canines de grands carnivores et des dents jugales de cheval utilisées comme retouchoirs de l'Aurignacien et du Moustérien du Sud-ouest de la France. Figure 1 - Distribution of large carnivores canines and horse jugal teeth used as retouchers in the Aurignacian and Mousterian of southwest France.

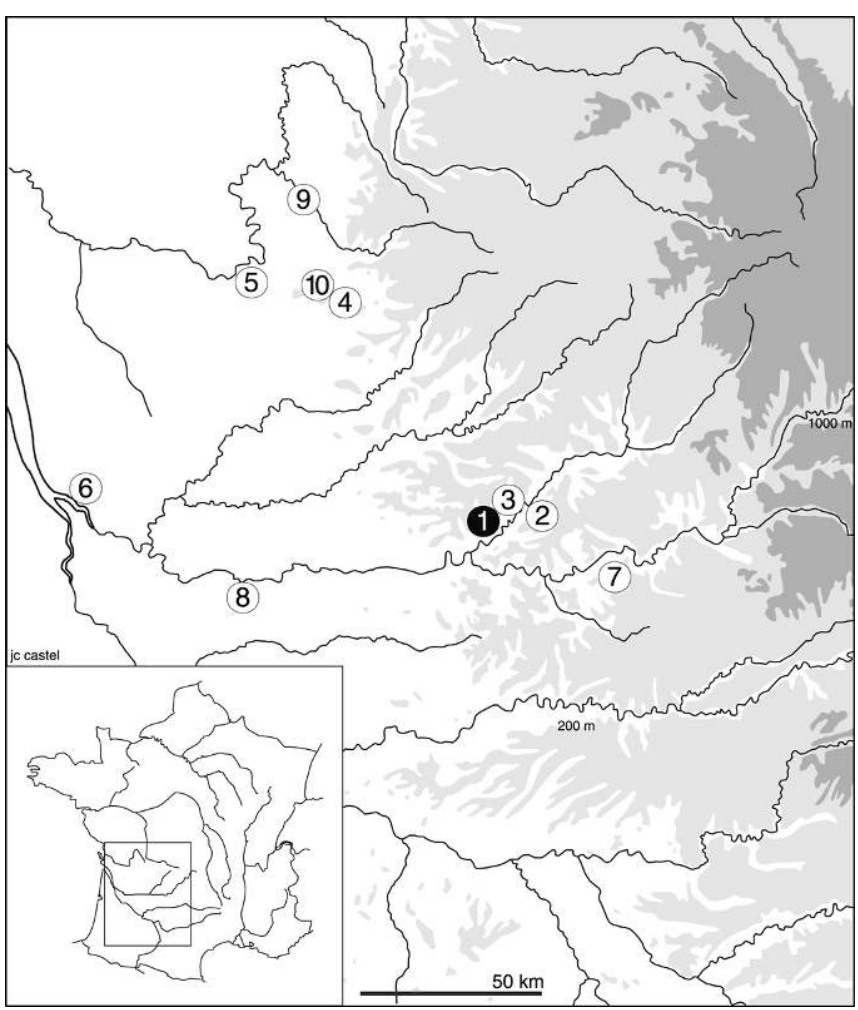

1 - La Ferrassie (Savignac-de-Miremont);

2 - La Souquette (Sergeac);

3 - Abri Pagès du Ruth (Tursac);

4 - Les Rois (Mouthiers) :

5 - Le Pont-Neuf (La Couronne);

6 - Pair-non-Pair (Prignac-et-Marcamps) ;

7 - Le Piage (Fajoles) ;

8 - Le Pigeonnier (Gensac):

9 - Artenac (Artenac) ;

10 - La Quina (Gardes-le-Pontaroux).

1 à 7 : dents de grands carnivores; 1 et 8 à 10 : dents de cheval ; 7 : dent de sanglier utilisée (information J.-G. Bordes) ; 9 et 10 : Moustérien.

1- 7: large carnivores teeth; 7, 8 and 10: horse teeth; 7: used wild boar tooth (information J.-G. Bordes); 9 and 10: Mousterian.

4 Comprenant un vaste abri et une petite grotte, le gisement La Ferrassie a été découvert à la fin du XIXème siècle (fig. 1). Une stratigraphie correspondant à une longue durée de mise en place et comprenant plusieurs assemblages archéologiques attribués à des techno-complexes paléolithiques différents (Moustérien, Châtelperronien, Aurignacien, 
Gravettien) a été précisée très tôt. De ce fait, le gisement a joué un rôle déterminant dans la connaissance de la chronologie du début du Paléolithique supérieur. Les différents niveaux archéologiques qui constituent l'Aurignacien ont ainsi historiquement servi de base à Denis Peyrony pour la subdivision de ce stade industriel en plusieurs phases, essentiellement à partir des caractéristiques de l'industrie osseuse (Peyrony 1933, 1934, 1937).

5 La faune provenant des fouilles de D. Peyrony a été examinée par E. Harlé (Peyrony 1934); celle issue des travaux de H. Delporte a été étudiée par F. Delpech $(1983,1984)$. Un inventaire récent des vestiges de la collection Peyrony, conservés au Musée National de Préhistoire (Madelaine, 1989), montre des dissemblances parfois importantes, dans les proportions des principaux Ongulés des différentes couches archéologiques, avec les décomptes réalisés par F. Delpech sur le matériel des fouilles Delporte (tabl. 1). Cependant, comme l'a constaté J.-P. Texier (2001), la stratigraphie du gisement est complexe et résulte d'importants mouvements sédimentaires d'origine périglaciaire. Dès lors, il devient difficile de déterminer quels ensembles fauniques sont les plus représentatifs des différentes phases culturelles.

Tableau 1- Inventaire partiel de la faune de La Ferrassie : collections Peyrony du Musée National de Préhistoire aux Eyzies (détermination S. Madelaine) et collection Delporte (Delpech 1983). Les fortes différences de représentation observées ne sont pas expliquées.

Table 1 - Partial inventory of the fauna of La Ferrassie: Peyrony's collections from the National Museum of Prehistory, Les Eyzies (determination by S. Madelaine) and Delporte collection (Delpech 1983). The significant differences in representation observed remain unexplained.

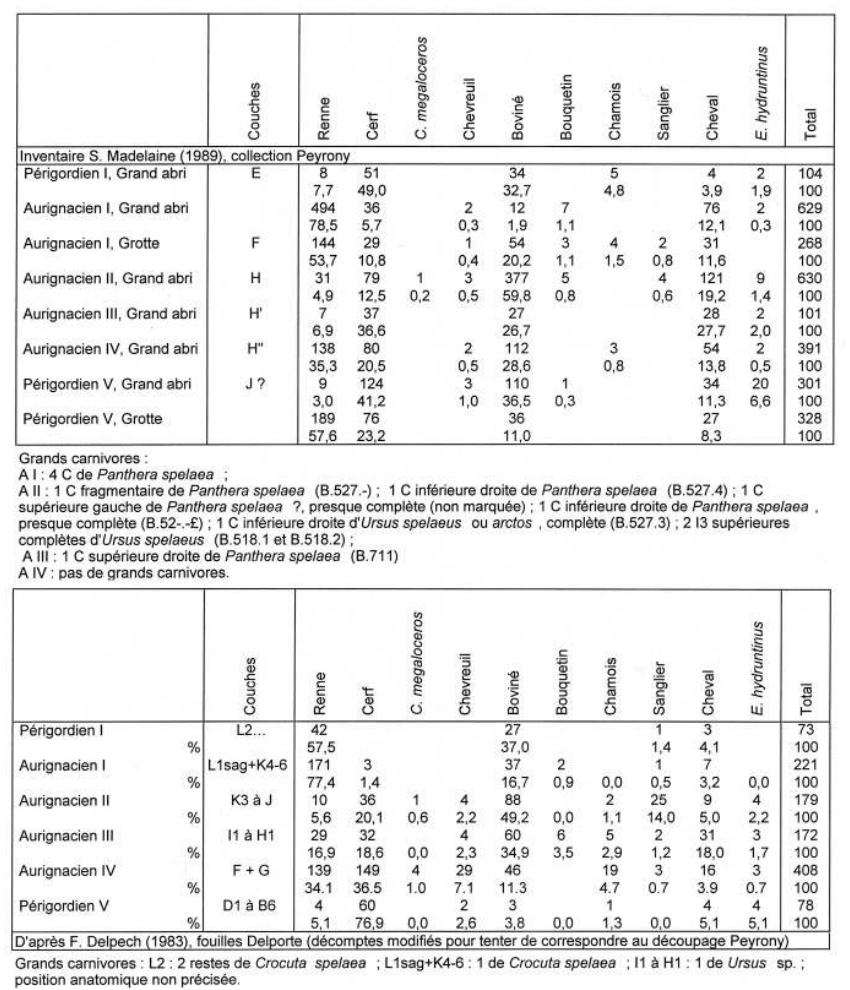

Quoi qu'il en soit, une unité relative règne dans les différents niveaux aurignaciens où les taxons principaux sont le Renne, le Cerf et les Bovinés. Ils sont accompagnés principalement par le Cheval et, dans certains niveaux, par le Sanglier. Le Chamois, le Bouquetin, le Chevreuil, le Megaceros et Equus hydruntinus sont parfois présents. Les carnivores les plus abondants sont le Renard, la Belette et le Loup. Lynx, Grand félin des 
cavernes, Hyène et Ours ne sont représentés que par un nombre extrêmement réduit de restes (Delpech 1983). Les morsures sont très rares sur les restes fauniques ordinaires mais un choix archéologique des pièces les mieux conservées a pu être opéré. Des Ongulés de toutes tailles ont constitué l'essentiel de l'acquisition alimentaire. Leurs restes osseux ont pu servir de supports à une partie de l'outillage en matières animales. Une majorité de dents, généralement complètes, accompagne également quelques petits os (carpe, tarse, phalanges) et des extrémités articulaires. La plupart des fragments de diaphyses présents dans les collections sont des retouchoirs. Ces derniers correspondent donc à une sélection, lors de la fouille, d'objets transformés, porteurs de stigmates caractéristiques d'une utilisation.

\section{2- L'échantillon analysé}

7 Les pièces présentées ici proviennent des fouilles de D. Peyrony dans la grotte et le grand abri. Elles se rapportent en majorité à l'Aurignacien I (couche F), sur lequel nous avons porté une attention particulière. Notre recherche a été plus rapide pour les niveaux plus récents. Par conséquent, l'inventaire des dents de chevaux et surtout celui des retouchoirs sur diaphyses, ne doit pas être considéré comme exhaustif. Outre les quatre canines de grands carnivores qui nous occupent ici en particulier, nous avons sélectionné deux dents de Cheval et 20 fragments de diaphyses de grands mammifères (tabl. 2, fig. 2). Entre leur découverte et les récentes restructurations des collections, les vestiges ont subi une usure par frottement et contact; elle est très perceptible sur les retouchoirs en os et dans une mesure moindre sur les dents. Ces dernières sont aussi victimes de phénomènes de dessication très classiques qui conduisent à leur éclatement progressif. Les dents de cheval d'un même niveau archéologique sont toutes disposées dans un même sachet ce qui provoque des chocs à chaque manipulation (ces dernières sont cependant rares). Par mesure de protection, les canines de carnivores ont été précocement isolées du reste du matériel et disposées ensemble dans une même boite en carton; elles ont pu toutefois s'entrechoquer. Même sur ces dernières, des traces post-dépositionnelles sont avérées et certaines zones utilisées n'ont pu être étudiées. La dent 435-1, très fragile, semble s'être "désintégrée" après la fouille; elle est reconstituée avec un mastic mais il existe de nombreux manques. Toutes les pièces repérées lors de notre examen ont été isolées dans des sachets transparents et, pour les dents, dans des boîtes en plastique transparent avec calage par de la mousse. 
Tableau 2 - La Ferrassie - corpus des vestiges étudiés. Les fragments de diaphyses 7 et 10 de notre inventaire sont en fait des os mordus (grooves).

Table 2 - La Ferrassie - corpus of studied remains. The diaphysis fragments 7 and 10 in our inventory are in fact gnawed bones (grooves).

\begin{tabular}{|c|c|c|c|c|c|c|}
\hline $\begin{array}{c}\text { Inventaire } \\
\text { MNP / } \\
\text { d'étude }\end{array}$ & Provenance & Espèce / taxon & Partie / état & $\mathrm{L}$ & $\mathrm{I}$ & $\mathrm{n}$ \\
\hline $\begin{array}{l}\mathrm{B}-4351 \\
\mathrm{~B}-4352 \\
\mathrm{~B}-435 \\
\mathrm{~B}-435 \\
\end{array}$ & $\begin{array}{l}\text { Aurignacien I - Grand abri } \\
\text { Aurignacien I - Grand abri } \\
\text { Aurignacien I - Grand abri } \\
\text { Aurignacien I - Grand abri } \\
\end{array}$ & $\begin{array}{l}\text { Panthera spelaea } \\
\text { Panthera spelaea } \\
\text { Panthera spelaea } \\
\text { Panthera spelaea? }\end{array}$ & $\begin{array}{l}\text { canine supérieure gauche, abimée reconstituée } \\
\text { canine supérieure gauche, couronne cassée } \\
\text { canine inférieure droite, complète } \\
\text { canine (supérieure gauche ou droite) racine }\end{array}$ & $\begin{array}{c}106,3 \\
98,7 \\
103,9 \\
73,4 \\
\end{array}$ & $\begin{array}{l}31,0 \\
31,5 \\
27,3 \\
30,8 \\
\end{array}$ & \\
\hline $\begin{array}{l}\text { sans } \\
\text { sans }\end{array}$ & $\begin{array}{c}\text { Grotte - Aurignacien I } \\
\text { Gd abri ou grotte - Aurignacien I ? }\end{array}$ & $\begin{array}{l}\text { Cheval } \\
\text { Cheval }\end{array}$ & $\begin{array}{l}\text { Molaire supérieure } 1-2 \text { droite } \\
\text { Prémolaire inférieure } 3-4 \text { gauche }\end{array}$ & $\begin{array}{l}60,5 \\
60,8 \\
\end{array}$ & $\begin{array}{c}31 \\
28,7\end{array}$ & $\begin{array}{c}1 \\
2 ?\end{array}$ \\
\hline 1 & sans indication & Grand mammifère & os long & 74 & 27 & 1 \\
\hline 2 & sans indication & Grand mammifère & tibia & 119 & 30 & 2 \\
\hline 3 & sans indication & Grand mammifère & fémur & 98 & 32 & 1 \\
\hline $120 / 4$ & Aurignacien I & Grand mammifère & os long & 159 & 53 & 1 \\
\hline 5 & Aurignacien I & Grand mammifère & os long & 72 & 27 & 1 \\
\hline 6 & Aurignacien III-IV & Cerf? & humérus? & 102 & 47 & 1 \\
\hline 8 & sans indication & Grand mammifère & os long & 92 & 40 & 1 \\
\hline 9 & sans indication & Boviné & fémur? & 161 & 41 & 1 \\
\hline 11 & Aurignacien I & Grand mammifère & fémur? & 117 & 46 & 1 \\
\hline 12 & Aurignacien I & Grand mammifère & os long & 69 & 35 & 1 \\
\hline 13 & Aurignacien I & Cerf & $\mathrm{mc}$ & 115 & 28 & 3 \\
\hline 14 & Grotte Aurignacien I-II & Grand mammifère & tibia & 148 & 45 & 2 \\
\hline 15 & Grotte Aurignacien I-II & Grand mammifère & tibia & 153 & 49 & 1 \\
\hline 16 & Aurignacien II & Grand mammifère & os long & 98 & 35 & 1 \\
\hline 17 & Aurignacien II & Grand mammifère & os long & 68 & 36 & 1 \\
\hline 18 & Aurignacien II & Grand mammifère & os long & 91 & 36 & 1 \\
\hline 19 & Aurignacien II & Grand mammifère & os long & 60 & 31 & 1 \\
\hline 20 & sans indication & Grand mammifère & humérus & 115 & 37 & 2 \\
\hline 21 & Aurignacien I & Grand mammifère & fémur & 102 & 50 & 3 \\
\hline 22 & Aurignacien II & Grand mammifère & os long & 111 & 35 & 1 \\
\hline
\end{tabular}


Figure 2 - La Ferrassie, Aurignacien I : dents utilisées comme retouchoirs, canines de grands carnivores et molaire de cheval (Photos P. Jugie, MNP). Échelle : $2 \mathrm{~cm}$

Figure 2 - La Ferrassie, Aurignacian I : teeth used as retouchers, large carnivores canines and horse molar (Photos P. Jugie, MNP). Scale : $2 \mathrm{~cm}$
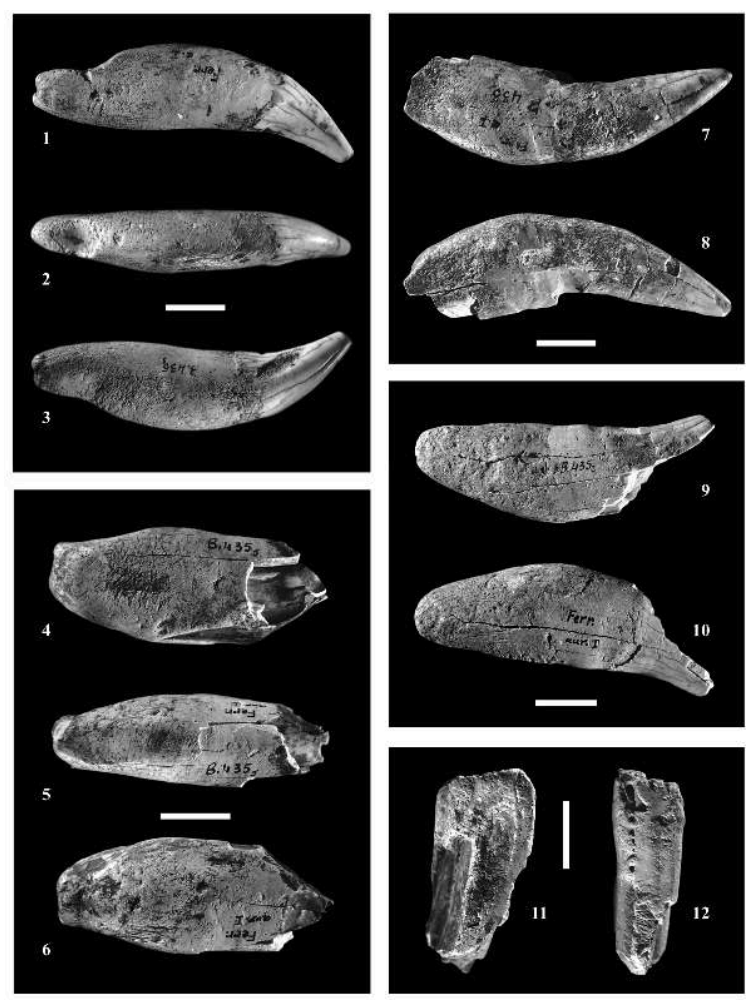

1 à 3 : B.435.3, Panthera spelaea, canine inférieure droite, dimensions : 103,9 × 27,3 mm; 1 : face interne, 2 : face antérieure, 3 : face externe. 4 à 6 : B.435.5, Panthera spelaea probable, canine supérieure gauche ou droite, $73,4 \times 30,8 \mathrm{~mm} ; 4$, face externe ou interne, 5 : face antérieure ou postérieure, $6:$ face interne ou externe. 7 et $8:$ B.435.1, Panthera spelaea, canine supérieure gauche, $106,5 \times 31,0 \mathrm{~mm} ; 7:$ face externe, 8 : face interne. 9 et 10 : B.435.2, Panthera spelaea, canine supérieure gauche, $98,7 \times 31,5 \mathrm{~mm} ; 9$ : face externe, 10 : face interne. 11 : Equus caballus, molaire supérieure 1 ou 2 droite, face mésiale ; $60,8 \times 28,7 \mathrm{~mm}$, facette utilisée : $44 \times 23 \mathrm{~mm}$. 12 : Equus caballus, prémolaire inférieure 3 ou 4 gauche, face mésiale, $60,8 \times 31 \mathrm{~mm}$, facette utilisée : $51,5 \times 16,5$ $\mathrm{mm}$.

7 to 3: B.435.3, Panthera spelaea, lower right canine, dimensions: $103,9 \times 27,3 \mathrm{~mm}$; 1: inner view, 2: anterior view, 3: exterior view. 4 to 6: B.435.5, probable Panthera spelaea, upper right or left canine, $73,4 \times 30,8 \mathrm{~mm}$; external or internal view, 5: anterior or posterior view, 6: internal or exterior view. 7 and 8: B.435. 1, Panthera spelaea, upper left canine, 106,5 × 37,0 mm; 7: exterior view, 8: interior view. 9 and 10: B.435.2, Panthera spelaea, upper left canine, $98,7 \times 37,5 \mathrm{~mm}$; 9: exterior view, 10: interior view. 17: Equus caballus, upper molar, right 1 or 2, mesial view: $60,8 \times 28,7 \mathrm{~mm}$, size of mesial side: $44 \times 23 \mathrm{~mm}$. 12: Equus caballus, lower premolar, left 3 or 4, mesial view: $60,8 \times 37 \mathrm{~mm}$, size of mesial side: $51,5 \times 16,5 \mathrm{~mm}$.

\section{2- Les retouchoirs de l'Aurignacien de La Ferrassie}

\section{1- Problématique}

8 L'intérêt de cette série réside dans la coexistence, au sein d'un même assemblage archéologique, d'artefacts - dents de grandes dimensions et diaphyses osseuses - de structure a priori différentes (au sens défini par Sigaut 1991) portant des stigmates d'utilisation apparemment semblables, au moins dans leur morphologie Ce fait archéologique n'est pas isolé, mais reste, dans l'état actuel de la recherche, uniquement observable dans quelques gisements aurignaciens comme ceux du Vogelherd IV (Hahn 1977; Taute 1965), Les Rois (Mouton et Joffroy 1958, p. 71, fig. 33), Le Pont-Neuf 
(Favraud 1907, p. 420, fig. 183), Pair-non-Pair (Cheynier 1963 p. 58 et 69). On peut ajouter à cette liste les canines de grands carnivores utilisées de quelques sites aurignaciens où les retouchoirs sur diaphyses ne sont pour l'instant pas signalés : Wildscheuer III (Hahn 1977), l'abri Pagès du Ruth (Leroy-Prost 1975, 1979, 1996), La Souquette (Mouton 1957 ; Castel et Madelaine., ce volume) ainsi que Le Piage (réexamen de J.-G. Bordes, mais voir aussi les figures de Champagne et Espitalié 1981). L'utilisation de dents jugales (prémolaires, molaires) de Cheval a été observée par A. Rutot (1908) dans la caverne d'Hastière en Belgique, mais à notre connaissance, ces vestiges ne sont pas décrits; Henri-Martin (1908), dans une note sur la communication de Rutot, les considère comme incertains. Un examen des séries moustériennes issues des fouilles de A. Debenath et A. Jelinek à la Quina indiquerait toutefois la présence de plusieurs de ces vestiges (D. Armand, com. pers.). D. Armand et A. Delagnes (1998, p. 207, fig. 1 et com. pers.) signalent également quatre dents jugales de Cheval utilisées dans le Moustérien d'Artenac. Il se trouve également une molaire inférieure utilisée dans l'Aurignacien de l'abri du Pigeonnier (Castel, en cours ${ }^{1}$ ).

9 Est-ce un hasard si c'est justement à l'Aurignacien ancien que ces pièces sur os et sur dents se retrouvent "fossilisées » dans les mêmes techno-complexes, alors que ce ne semble plus être le cas pour les ensembles industriels postérieurs ? En effet, ce moment du Paléolithique supérieur qu'est l'Aurignacien ancien correspond, d'une part, à la mise en place d'une industrie sur matières dures animales (Leroy-Prost 1975, 1979; Liolios 1992, 1999) et d'autre part, à l'émergence d'une symbolique où les images de l'Ours et $\mathrm{du}$ Lion sont particulièrement prégnantes comme en attestent, par exemple, les figurations pariétales de la grotte Chauvet (Vallon-Pont d'Arc, Ardèche) (Clottes et al., 2001 ; Morel et Garcia 2002), la statuette à la fois léonine et anthropomorphe de Hohlenstein-Stadel (Bade-Württemberg, Allemagne) ou les rondes-bosses d'Ursidé et de Félin du Vogelherd (Bade-Württemberg, Allemagne) (Bosinski 1990). Notons enfin la mise en fonction des canines de ces mêmes animaux comme objets de parure, portés ou non en suspension, retrouvés à l'unité dans les gisements de Combe-Saunière et de l'abri Pataud, par exemple (Brooks 1995 ; Chauvière 1997²).

10 C'est, à notre avis, dans cet environnement technique et symbolique, spécifique de l'Aurignacien, que des hypothèses peuvent être formulées pour tenter d'expliquer l'anomalie technologique mentionnée plus haut. Toutefois, les données nécessaires ne peuvent être réunies si, comme cela a été le cas jusqu'à présent, la dissociation par catégories de matériel - os d'un côté, dent de l'autre - reste la règle. L'approche contextuelle permet seule de pallier cet éclatement des analyses en réintégrant la discussion dans le champ global des matériaux osseux. Dès lors, l'ensemble des observations pourra être envisagé dans une perspective méthodologique et interprétative de même niveau qui mettra en évidence contraintes physiques et mécaniques de la matière avant d'inférer des comportements humains.

\section{2- Méthodologie}

11 Outre une description précise de ces pièces, conforme à celle préconisée (cf. PatouMathis et al. 2002 ; Vincent 1993), nous avons opté pour une approche résolument technique et fonctionnelle de ce matériel de La Ferrassie, en essayant toutefois de remonter jusqu'à la gestuelle technique qui a pu produire les stigmates observables. L'outil conceptuel utilisé est celui de la châne opératoire tel que l'a définie A. Leroi- 
Gourhan (1943) et qui permet d'ordonner, à l'échelle du geste technique, la succession chronologique des différents stigmates d'origine anthropique.

Nous avons également utilisé notre propre référentiel expérimental, utilisable à l'échelle macroscopique et sous loupe binoculaire (grossissement jusqu'à x 80), pour sérier les observations effectuées sur le matériel archéologique.

\section{3- L'utilisation technique des dents de grands mammifères}

Les dents utilisées se répartissent en quatre canines de grands carnivores et deux dents jugales de Cheval. Parmi les premières, trois sont attribuables incontestablement à Panthera spelaea. La canine publiée par Peyrony, et qui est la plus complète, avait été attribuée à Ursus speleaus (Capitan et Peyrony 1912 ; Peyrony 1934) puis, après révision par Mouton (1957) à Felis spelaeus qui équivaut maintenant à Panthera spelaea. La quatrième, dont la couronne est brisée, se rapproche plus de Panthera spelaea que de Ursus spelaeus. Le Cheval a fourni une molaire supérieure ainsi qu'une prémolaire inférieure dont les stigmates sont plus douteux (fig. 2).

\section{1- Les dents de Lion et d'Ours}

L'état fragmentaire des canines utilisées de La Ferrassie nous a conduit à une observation détaillée, axée sur la distinction entre les canines des félidés et celles des ursidés, apparemment peu fréquente, voire absente de la littérature. La présence de sillons longitudinaux sur la couronne des canines de félins semblait être le seul caractère cité pour distinguer ce taxon. Or, l'observation de ce caractère se fait surtout sur les canines supérieures et dépend de la bonne conservation de l'émail fréquemment détérioré par de nombreuses fissures. Suite à une comparaison détaillée de canines facilement déterminables grâce à leur bon état de conservation, nous proposons de présenter les critères distinctifs des canines supérieures et inférieures des félidés et des ursidés (tabl. 3). Certains caractères sont souvent cités isolément par les différents spécialistes de ces familles (par exemple Bonifay M.-F. 1971; Ballesio 1975 ; Suire 1968, 1970; Koby 1952): notre comparaison nous a permis de découvrir des points discriminants nouveaux. Le matériel de référence provient des collections du Musée national de Préhistoire des Eyzies : les ours (des cavernes et bruns) sont issus des sites de Font-de-Gaume (fouilles Prat), du Régourdou (fouilles Bonifay), de La Ferrassie (fouilles Peyrony) et du Clos-du-Prieur (fouilles Madelaine); les félins (Panthera onca gombaszoegensis et Panthera spelaea) proviennent des sites de L'Escale, Lunel-Viel, Le Régourdou (fouilles Bonifay), La Ferrassie (fouilles Peyrony) et du Clos-du-Prieur (fouilles Madelaine). 
Tableau 3 - Critères de distinction des canines des Félidés et des Ursidés (en italique les caractères non décrits jusqu'alors).

Table 3 - Criteria for distinguishing the canines of Felidae and Ursidae (the characters until now unidentified are indicated in italics).

\begin{tabular}{|c|c|c|}
\hline Canines supérieures & Panthera & Ursus \\
\hline Vue externe & $\begin{array}{l}\text { - Double sillon longitudinal, situé dans la } \\
\text { moitié supérieure de la couronne, } \\
\text { délimitant un bourrelet. }\end{array}$ & - Absence de sillon \\
\hline \multirow[t]{2}{*}{ Vue interne } & $\begin{array}{l}\text { - Double sillon longitudinal, situé dans la } \\
\text { moitié supérieure de la couronne. }\end{array}$ & - Absence de sillon \\
\hline & $\begin{array}{l}\text { - Crête antéro-interne (ou mésio- } \\
\text { linguale), souvent bien marquée, } \\
\text { proche du bord antérieur, pouvant se } \\
\text { terminer par un bourrelet au collet. } \\
\text { - Usure possible au niveau de la crête. }\end{array}$ & $\begin{array}{l}\text { - Crête antéro-interne peu marquée. } \\
\text { - L'usure se fait le long de la crête et } \\
\text { surtout obliquement sur la face } \\
\text { interne, proche du collet. }\end{array}$ \\
\hline Vue interne ou externe & $\begin{array}{l}\text { - Profil postérieur de la couronne peu } \\
\text { arqué (proche de la verticale). }\end{array}$ & - Profil de la couronne arqué. \\
\hline $\begin{array}{l}\text { Vue postérieure } \\
\text { (= distale) }\end{array}$ & $\begin{array}{l}\text { - Crête distale très coupante, parsemée } \\
\text { fréquemment de petites stries } \\
\text { horizontales sur toute sa hauteur (si non } \\
\text { usée). } \\
\text { - Cette crête est située sur une verticale } \\
\text { au milieu de la couronne. } \\
\text { - L'usure distale se fait sur l'apex et le } \\
\text { long de la crête distale. }\end{array}$ & $\begin{array}{l}\text { - crête distale moins nette. } \\
\text { Absence de stries perpendiculaires. } \\
\text { - Cette crête est située sur le tiers } \\
\text { externe de la couronne. } \\
\text { - Pas d'usure sur la crête distale. }\end{array}$ \\
\hline \multicolumn{3}{|l|}{ Canines inférieures } \\
\hline Vue externe & $\begin{array}{l}\text { - } 1 \text { seul sillon longitudinal, situé dans la } \\
\text { moitié supérieure de la couronne. } \\
\text { - Usure fréquente, oblique, partant de la } \\
\text { crête distale vers le collet. }\end{array}$ & $\begin{array}{l}\text { - Pas de sillon. } \\
\text { - Pas d'usure observée } \\
\text { (mais échantillon faible). }\end{array}$ \\
\hline Vue interne & $\begin{array}{l}\text { - Crête interne (ou linguale) et non } \\
\text { antéro-interne (cas des C.sup) se } \\
\text { terminant par un bourrelet net au niveau } \\
\text { du collet. } \\
\text { - Usure oblique au dessus du bourrelet. }\end{array}$ & $\begin{array}{l}\text { - Crête antéro-interne peu marquée, } \\
\text { plus antérieure que chez les Félins, } \\
\text { et sans bourrelet net au collet. } \\
\text { - Usure parfois identique aux } \\
\text { Félidés. }\end{array}$ \\
\hline $\begin{array}{l}\text { Vue postérieure } \\
\text { (= distale) }\end{array}$ & $\begin{array}{l}\text { - Crête verticale très marquée, } \\
\text { coupante, ayant fréquemment des } \\
\text { stries horizontales (comme les } C \text {. sup). }\end{array}$ & - Crête verticale peu marquée. \\
\hline Vue externe ou interne & \multicolumn{2}{|c|}{ Profils arqués très similaires. } \\
\hline Vue antérieure & \multicolumn{2}{|c|}{ Très similaires. } \\
\hline
\end{tabular}

Même si ce matériel n'est pas très important quantitativement, il nous a permis d'identifier trois des quatre pièces de façon certaine. Au sein de ces deux familles, la variabilité interspécifique et intraspécifique (due, entre autres, au dimorphisme sexuel) est tellement importante (particulièrement sur les racines) que nous n'avons pas tenu compte des différences dans les dimensions, qui apparaissent trop subjectives sur une dent isolée (sur un échantillon assez grand, on constate toutefois que les canines supérieures sont plus grandes et possèdent une racine plus globuleuse). Il en est de même de l'aspect plus sinueux, en vue antérieure ou postérieure, des canines inférieures par rapport aux canines supérieures, ces dernières étant plus rectilignes. Bien qu'il soit plus visible chez les ursidés, ce caractère peut donner lieu ici à des appréciations différentes suivant les observateurs.

- [50 [ Canine B.435.1 : canine supérieure gauche de Panthera. Malgré quelques fissures de l'émail, les doubles sillons isolant un bourrelet sont très nets sur les deux faces. La crête distale, usée tout le long, est peu visible, particulièrement au niveau de l'apex. Cette crête verticale est exactement au milieu de la couronne. De profil, le bord postérieur de la couronne est proche de la verticale. La crête mésio-distale et le bourrelet sont peu marqués à cause de l'usure.

- Fon Canine B.435.2 : canine supérieure gauche de Panthera. Malgré l'énorme cassure de la couronne, une partie du sillon longitudinal est visible sur chaque face. La crête distale, usée, sépare verticalement la couronne. L'orientation de la couronne en vue postérieure permet de deviner la latéralisation.

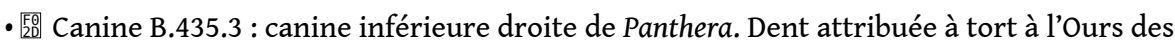
Cavernes (Capitan et Peyrony 1912 ; Peyrony 1934) puis rendue à Panthera par Mouton 
(1957). Elle est entière et possède un seul sillon sur sa face externe. Cette face est usée fortement en oblique. La face linguale est marquée d'une crête interne (et non antérointerne comme sur les canines supérieures), ponctuée d'un bourrelet très net au niveau du collet. Bien qu'usée à l'apex et près du collet, la crête distale est bien marquée et striée par des petites entailles horizontales.

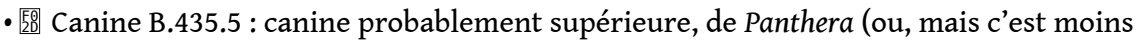
vraisemblable, d'Ursus). Seule une partie de la racine est conservée. Les dimensions et l'aspect globuleux, par comparaison, indiqueraient plutôt une canine supérieure : la forme d'ensemble est très proche de la B.435.2.

Figure 3 - La Ferrassie - détail des plages d'utilisation de B.435.1 (voir aussi 7.3 et 2.8) ; informations plus détaillées dans le tableau 4.

Figure 3 - La Ferrassie, close-up of the utilized zones of B.435.1 (see also 7.3 and 2.8); more detailed information in table 4.

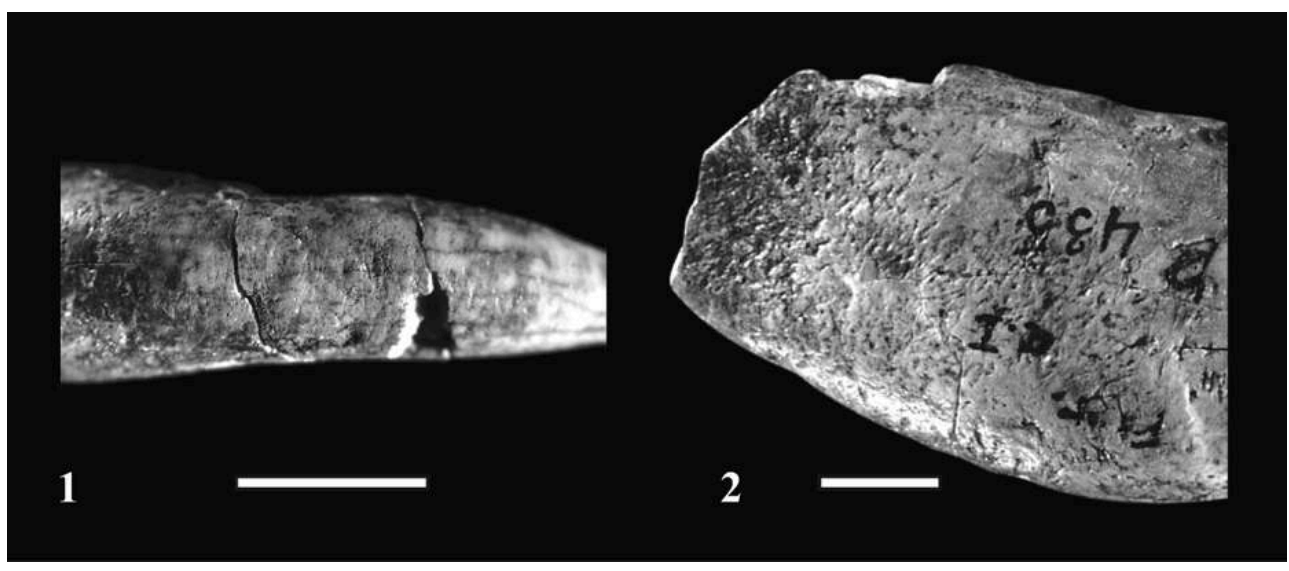

$1:$ face antérieure $; 2:$ face externe. Échelle $=1 \mathrm{~cm}$ 1: anterior view; 2: exterior view. Scale $=1 \mathrm{~cm}$. 
Figure 4 - La Ferrassie - détail de la plage d'utilisation de B.435.2 située sur la face antérieure (voir aussi 2.9 et 2.10). Échelle $=1 \mathrm{~cm}$.

Figure 4 - La Ferrassie, close-up of the utilized zone on anterior side of B.435.2 (see also 2.9 and 2.10). Scale $=1 \mathrm{~cm}$.

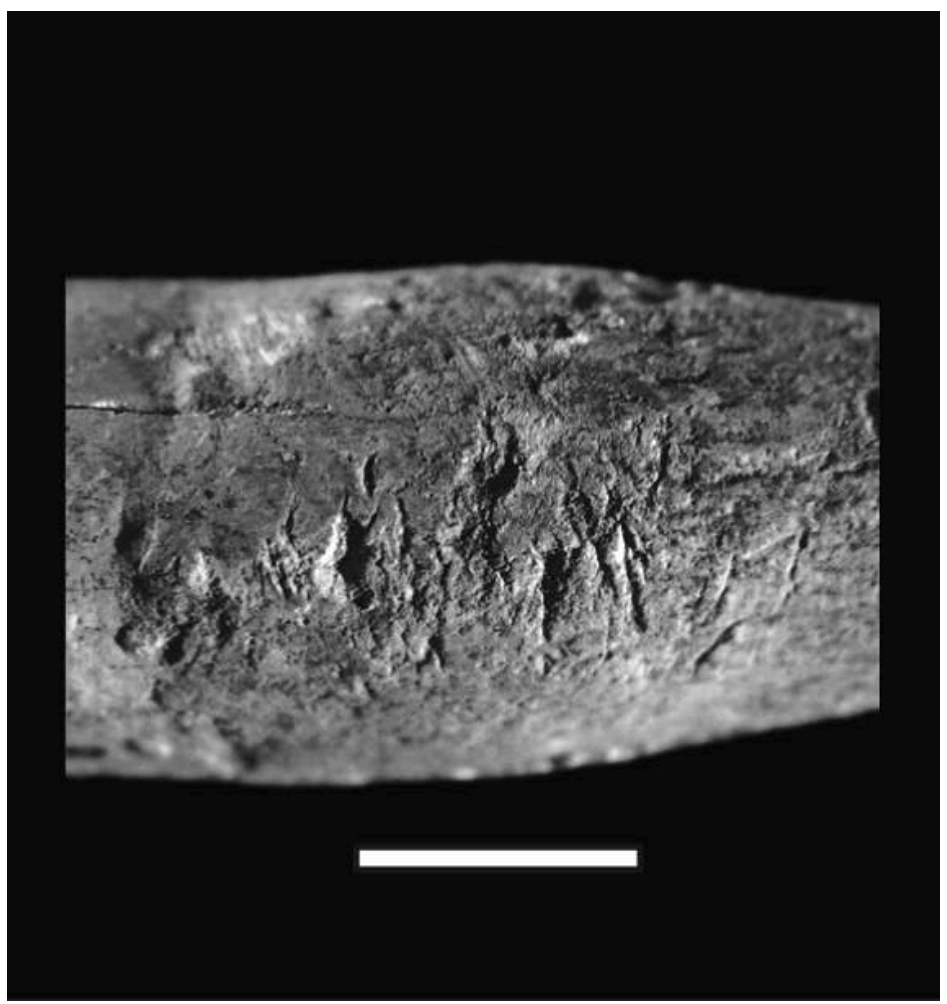

Figure 5 - La Ferrassie - détail des plages d'utilisation de B.435.3 (voir aussi 2.1, 7.1 et 7.2). Figure 5 - La Ferrassie, close-up of the utilized zones of B.435.3 (see also 2.1, 7.1 and 7.2).

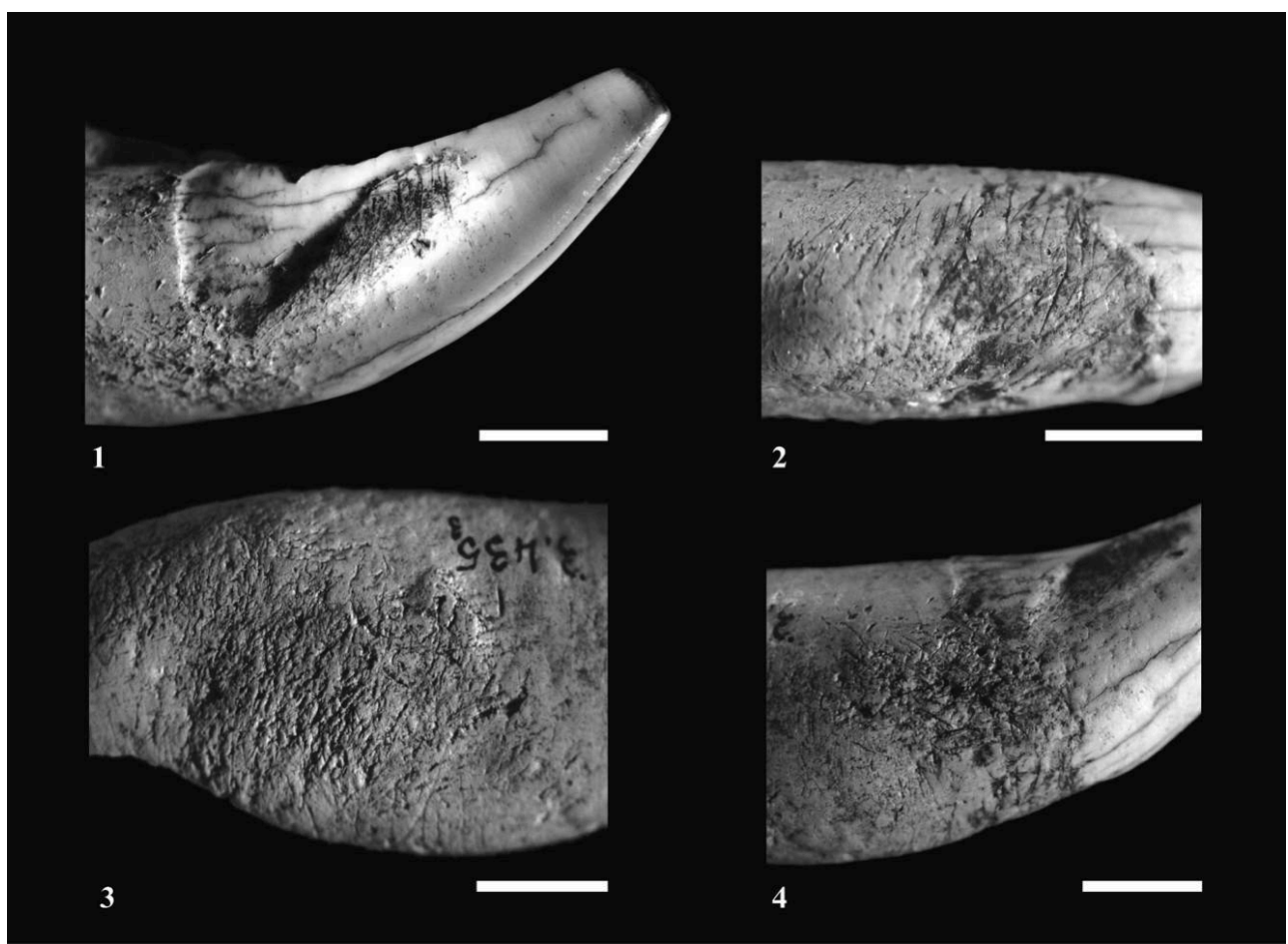

1 : rainure d'usure naturelle de la couronne ; 2 : face antérieure ; 3 et $4:$ face externe. Échelle $=1 \mathrm{~cm}$. 1: crown natural wear groove; 2: anterior view; 3 and 4: exterior view. Scale $=1 \mathrm{~cm}$. 
Figure 6 - La Ferrassie - détail de la plage d'utilisation de B.435.5 située sur la face antérieure (voir aussi $2.4,2.6$ et 7.2 ). Échelle $=1 \mathrm{~cm}$.

Figure 6 - Ferrassie, close-up of the utilized zone on anterior side of B.435.5 (see also 2.4, 2.6 and 7.2). Scale $=1 \mathrm{~cm}$.

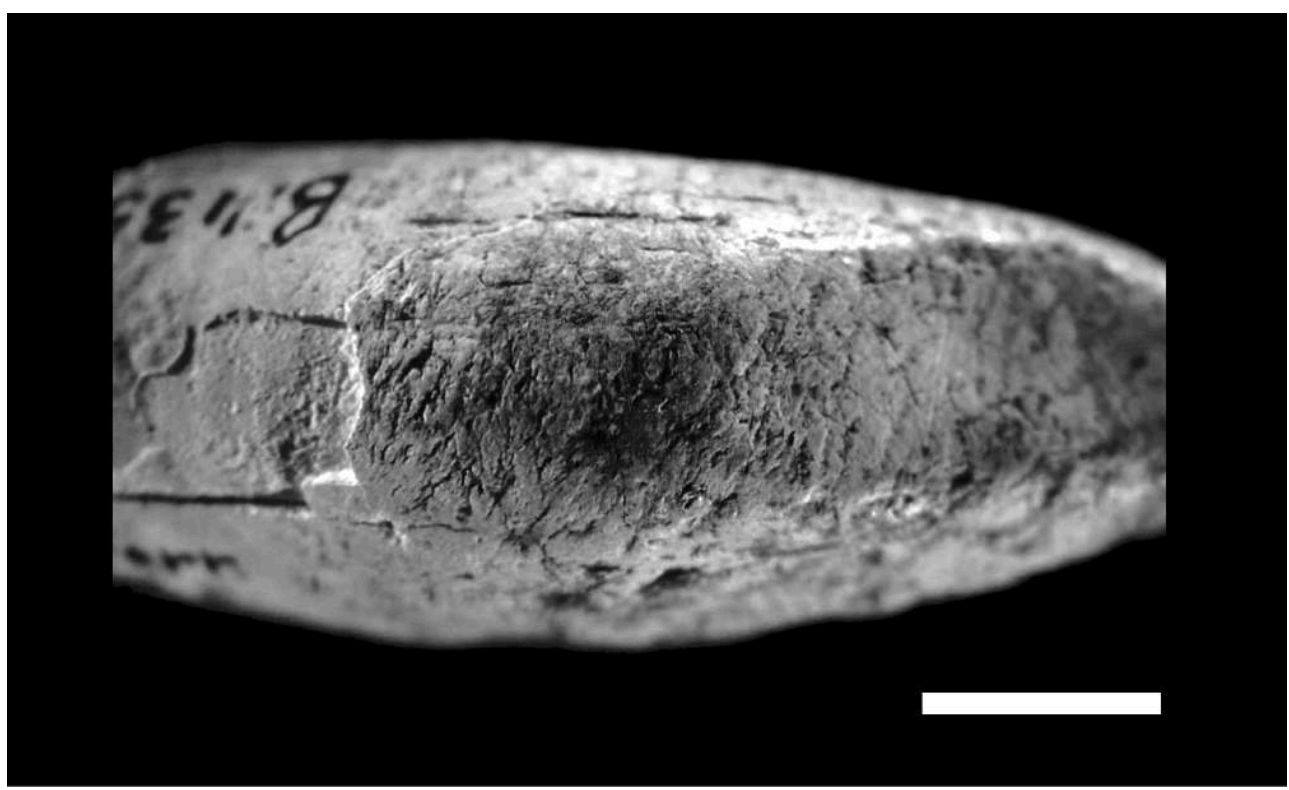

Tableau 4 - Description des stigmates et des plages de stigmates des différents types de retouchoirs de La Ferrassie.

Table 4 - Description of the traces and zones of traces of the different types of retouchers from La Ferrassie.

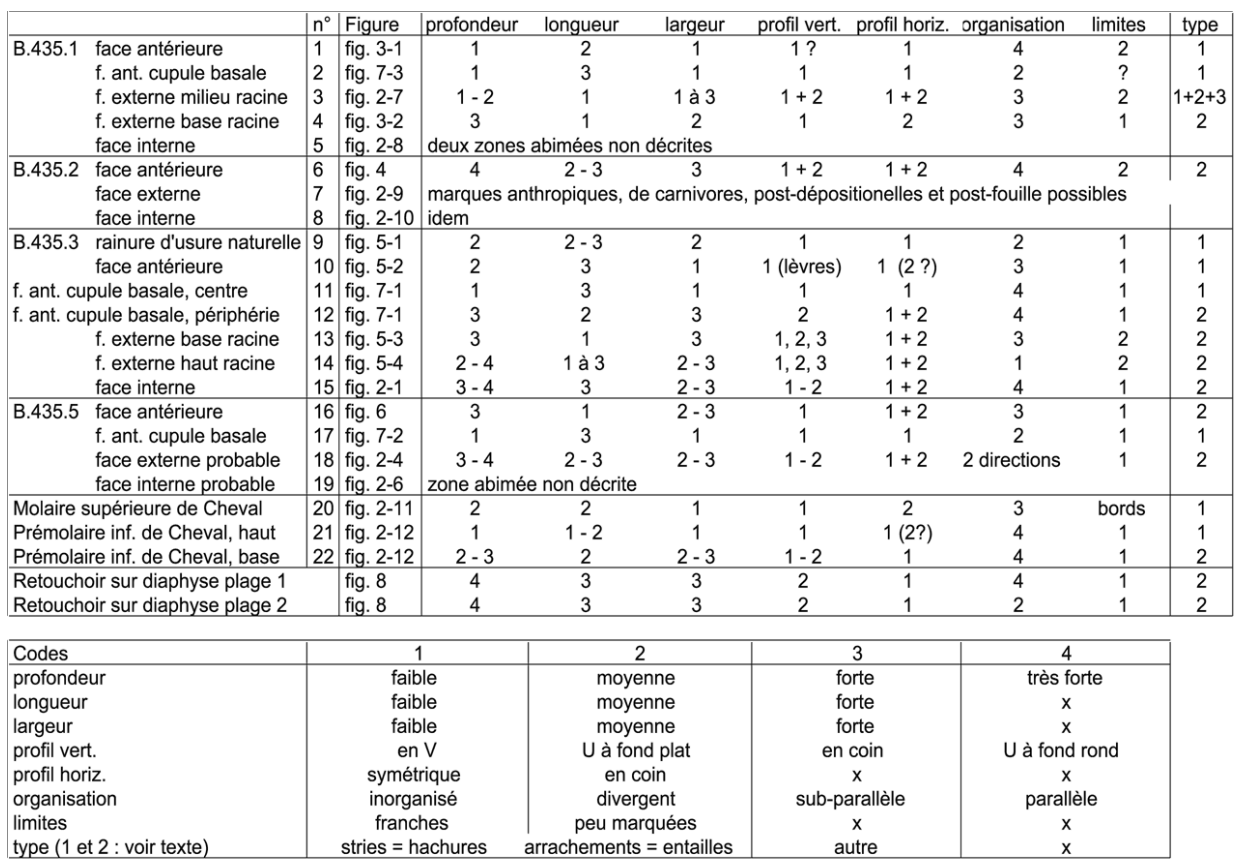

Les félins comme les ours ne sont représentés dans l'Aurignacien de La Ferrassie que par des restes dentaires et principalement des canines. Que celles-ci portent ou non des traces de modifications intentionnelles, elles peuvent être assimilées à une récupération de biens à forte valeur symbolique. Les canines de félins utilisées 
constituent, en outre, la totalité des vestiges de grands carnivores introduits dans l'Aurignacien I (tabl. 1 ; fouilles Peyrony, collections du MAN et du MNP).

\subsection{2- Acquisition}

17 Les molaires de Cheval de La Ferrassie peuvent avoir été prélevées dans le «stock dentaire " introduit dans le site, en même temps que des carcasses destinées à la consommation alimentaire, par le biais d'une activité cynégétique (Delpech 1983, 1984). En revanche, les canines de Lion posent problème quant à leurs modalités d'acquisition. Dans l'Aurignacien de La Ferrassie, les dents de grands carnivores sont au nombre d'une dizaine dont une majorité de canines (tabl. 1). L'absence presque complète de dents jugales et de restes post-crâniens semble indiquer qu'elles ont été recueillies et transportées pour des besoins uniquement techniques et symboliques. Même s'il existe un tri important des vestiges fauniques, les éléments remarquables du squelette des grands carnivores auraient sans doute été récoltés, ne serait-ce que pour satisfaire la curiosité des paléontologues de l'époque. L'acquisition de ces canines de grands fauves, qui restent rares tout au long du Paléolithique supérieur, est une phase de la chaîne opératoire mal documentée pour les temps paléolithiques et l'Aurignacien n'échappe pas à la règle. Rappelons que les niveaux gravettiens du gisement d'Hohle Fels ont livré le seul témoignage direct d'une chasse d'un grand carnivore par l'intermédiaire d'un impact de projectile resté fiché dans une vertèbre d'Ursus spelaeus (Münzel et al. 2001). L'extraction pratiquée au détriment de squelettes fossiles est en revanche documentée en milieu spéléologique comme au Tuc-d'Audoubert (Bégouen et Breuil 1958 ; Bégouen et Clottes 1982).

\subsection{3- De la transformation à l'utilisation}

Une seule canine est anatomiquement complète (B.435.3) alors que les autres sont brisées au niveau de la couronne, au niveau collet-couronne ou à la racine. Toutefois, cette fragmentation ne gêne en rien la lecture des stigmates d'origine anthropique car la conservation des états de surface est satisfaisante.

\section{Diversité des stigmates d'utilisation}

Les stigmates de l'utilisation anthropique sont préférentiellement localisés à la racine des canines de Félin ou occupent toute une face des dents de Cheval (fig. 2). Ils investissent, de façon plus ou moins marquée, trois faces de la dent sous forme de plages plus ou moins bien délimitées; la face postérieure n'est pratiquement pas utilisée. Leur présence au niveau de la couronne n'a été observée que dans un cas (B. 435.3), dans la rainure d'usure naturelle, sur la face distale (fig. 5, $\mathrm{n}^{\circ} 1$ ). Les plages d'utilisation examinées sur les dents, au nombre d'une vingtaine, se répartissent classiquement en deux groupes principaux (tabl. 4).

- [50 Type 1 : les arrachements de matière ou entailles ( $c f$. tabl. 4 et figures $3.2,4,7.1,8$, etc.). Il s'agit en général de dépressions profondes, larges et irrégulières.

- 医 Type 2 : les incisions de type «stries » ou hachures (tabl. 4 et figures 3.1, 7.3, 5.1, 7.1 etc.). Il n'y a pas d'enlèvement de matière mais compression, dans des stries rectilignes, régulières, à section en « $\mathrm{V}$ » : en ce sens, elles sont à rapprocher des stries de découpes et non des stries d'abrasion ou de raclage pour lesquelles il y a enlèvement de matière. 
Stries ou arrachements peuvent avoir un profil (horizontal ou vertical) asymétrique et donc former des coins (dépressions à profil triangulaire allongé dans un plan horizontal ou vertical). Mais, ce type de stigmates reste minoritaire dans les plages examinées (à la différence de certains retouchoirs sur diaphyses du Paléolithique supérieur (Castel 1999). Des lèvres peuvent être observées ponctuellement. L'organisation des marques n'est pas toujours décelable et les limites des plages ne sont pas très nettes. Ces deux caractéristiques permettent de distinguer les retouchoirs sur canines de ceux qui sont réalisés sur diaphyses.

21 Les différents types de stigmates peuvent être superposés. Nous n'avons pas quantifié l'intensité d'utilisation. Lorsqu'ils sont abondants, et qu'il s'agit d'arrachements, des " cuvettes » exactement localisées peuvent être observées. Les méplats observés à la surface interne et externe des canines B.435.1 (fig. 2.9, 2.10) et B.435.3 (fig. 2.1, 2.3) sont, de toute évidence, d'origine anthropique car ils ne se retrouvent pas sur les dents non travaillées.

Outre la fréquence des plages à incisions (hachures), qui ne se retrouvent que rarement sur les retouchoirs sur diaphyses, ce qui retient l'attention dans cette comparaison, c'est la diversité des stigmates observés, qu'il s'agisse d'arrachements ou d'incisions. Certaines plages à arrachements sont tout à fait semblables à celles des retouchoirs sur diaphyses (plages $n^{\circ} 4,12,15,18$, respectivement fig. 3.2, 7.1, 2.1, 2.4), d'autres montrent au contraire des limites peu marquées ( $n^{\circ} 3$ et 6 , fig. 2.7, 4), des changements de direction ( $n^{\circ} 9$ et 14 , fig. $\left.5.1,5.4\right)$ ou d'intensité d'utilisation ( $n^{\circ} 3$, fig. 2.7).

Les plages à stries (hachures) sont préférentiellement situées sur les faces postérieures des canines et dans des zones naturellement concaves ; on les trouve également sur les dents de Cheval. Les plages ont souvent des limites plus nettes et une plus grande homogénéité dans l'organisation directionnelle des stigmates. En revanche, on observe une plus grande diversité dans les caractéristiques dimensionnelles des incisions (ce qui peut aussi résulter des caractéristiques mécaniques variables des différentes parties des dents).

\section{Mise en forme de la racine des canines de Panthera}

Une dépression peut être observée sur la face antérieure, près de l'apex de la racine de B.435.3 (fig. 2.2, 7.1) et de B.435.5 (fig. 2.4 et 7.3) ; la racine de B.435.1 présente, sur la face antérieure, une amorce de dépression similaire mais elle est brisée (fig. 2.8 et 7.2). Sur B.435.3, cette dépression pourrait correspondre à l'enlèvement d'un éclat, détaché par percussion lancée sur le maxillaire pour en extraire la canine. Mais la présence d'un négatif de bulbe traduit une percussion lancée depuis la face interne, ce qui est possible pour des vestiges fossiles dont la mandibule ou le crâne sont déjà fragmentés (fig. 7). Cet enlèvement pourrait être une conséquence accidentelle de l'extraction des canines de grands carnivores, quelle que soit l'utilisation envisagée et, par conséquent, se retrouver sur une certaine proportion des dents. Cependant, ce genre d'enlèvement n'apparaît ni sur les canines percées ni sur la majorité des canines utilisées. Au contraire, la restriction de ce type de traces à un nombre limité de retouchoirs (La Ferrassie, Les Rois et peut-être une des dents du Pont Neuf et une de Vogelherd) conduit à envisager son caractère intentionnel et lié à une utilisation particulière. Pour trancher définitivement cette question, il serait nécessaire de réexaminer, dans son ensemble, la population de canines sélectionnées par l'homme à des fins techniques ou 
symboliques. La profonde similitude entre les pièces de La Ferrassie (MNP B.435.3, B. 435.5 et sans doute B.435.1) et des Rois (MAN R5233b) ainsi que la forte densité de traces (stries - hachures) présentes dans la dépression, nous incitent à penser que cet enlèvement est lié à l'utilisation spécifique de cette partie de la racine: création accidentelle d'un enlèvement en cours d'utilisation ou, plus sûrement, aménagement spécifique avant utilisation. Cette hypothèse d'un façonnage antérieur à la formation des stigmates, déjà proposée par Mouton (1957), réapparaît à la suite de nos observations, cela malgré l'absence de traces visibles de cette phase opératoire, du moins selon nos focales d'observation. La superposition des stigmates d'utilisation pourrait avoir oblitéré toute action technique préalable. L'émoussé des bords de cette dépression, sans qu'il soit véritablement interprétable d'un point de vue technique, permet de confirmer l'utilisation spécifique et intense de cette surface concave. La surface concave de la racine de B.435.5 présente également des stigmates d'utilisation ; sur B.435.1, la dépression, qu'elle soit intentionnelle ou non, porte également des traces identiques (fig. 7.2).

Figure 7 - La Ferrassie - dépressions et stigmates d'utilisation sur la base des racines : 1 : B.435.3, face antérieure ; $2:$ B. $435.5 ; 3:$ B.435.1, face antérieure. Échelle $=1 \mathrm{~cm}$.

Figure 7 - La Ferrassie - depressions and use traces on the base of the roots: 1: B.435.3, anterior surface; 2: B.435.5; 3: B.435.1, anterior surface. Scale $=1 \mathrm{~cm}$.

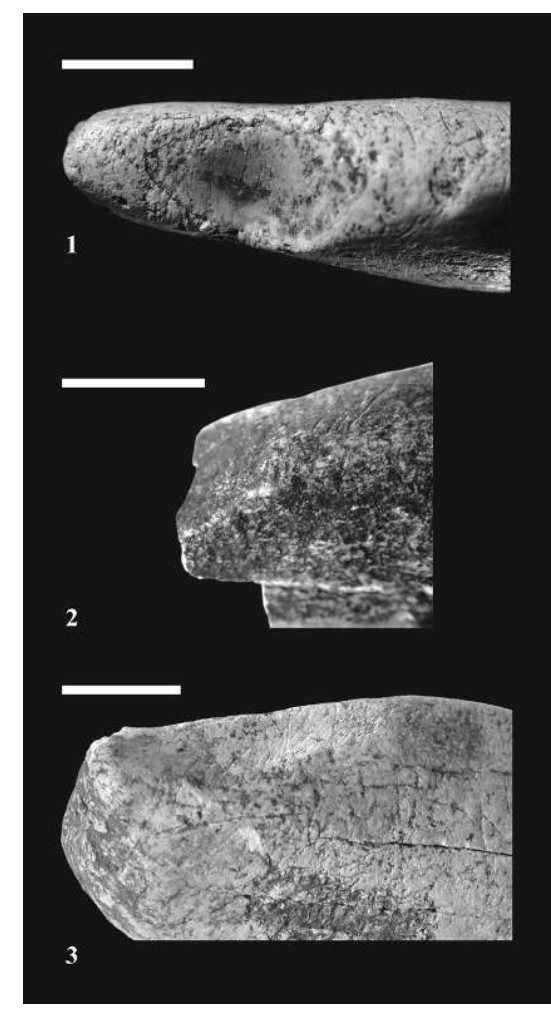

\section{Utilisation des surfaces concaves}

Sur la dent B.435.3, on observe l'utilisation de la surface d'usure issue du contact entre canine supérieure et inférieure (figure 5.1). Elle est à mettre en parallèle avec l'aménagement puis l'utilisation intense des dépressions, sur la base de la racine de trois dents sur quatre. Les stigmates sont constitués essentiellement de fines stries parallèles perpendiculaires à l'allongement des dents. Cette sélection de surfaces 
parfois relativement concaves et leur mode d'utilisation sont le résultat d'objectifs techniques spécifiques nécessairement différents de ceux satisfaits par l'utilisation classique des surfaces plano-convexes des dents, des diaphyses et des autres supports sur os en général. L'originalité de ces canines utilisées se situe notamment à ce niveau. Toutefois, ces types d'utilisation et de traces ne sont pas présents sur toutes les canines observées. La majorité semble avoir fonctionné de façon similaire aux retouchoirs sur os, mais un examen approfondi de l'ensemble de la population de dents utilisées est nécessaire. Ce n'est qu'alors qu'il sera possible de déterminer s'il s'agit d'une caractéristique technique spécifique de l'ensemble de cette catégorie de vestiges ou uniquement d'un sous-ensemble.

\section{Les dents de Cheval} Peyrony et les fouilleurs de la première moitié du XXe siècle (fig. 8). Il s'agit donc de pièces caractéristiques, faciles à identifier, et non d'une population d'outils avec toute sa variabilité dans la morphologie des volumes ou dans l'intensité d'utilisation. Les supports sont des diaphyses d'os longs de grands Ongulés. Il est possible qu'il y en ait eu sur diaphyses d'os longs de Renne, mais ils n'ont vraisemblablement pas été recherchés. En revanche, étant donné les connaissances de l'époque (Baudouin et al. 1907 ; Henri-Martin 1907-1910), s'il y en avait eu sur des phalanges d'Equidés ou des distum d'humérus de grands Ongulés, ils auraient certainement été vus et isolés. $\mathrm{Ne}$ pouvant accéder aux notions de choix des supports, de fréquence de cet outil dans l'ensemble archéologique et d'intensité d'utilisation, nous nous bornerons à décrire la morphologie des objets, la localisation des plages d'utilisation et à évoquer les 
caractéristiques des stigmates. Enfin, signalons que de nombreux retouchoirs sur diaphyses ont été observés dans les niveaux moustériens et gravettiens de La Ferrassie.

Figure 8 - La Ferrassie - Retouchoir sur diaphyse d'os long de grand Ongulé. Échelle $=2 \mathrm{~cm}$. Figure 8 - La Ferrassie - Retoucher on the diaphysis of a large Ungulate long bone. Scale $=2 \mathrm{~cm}$.

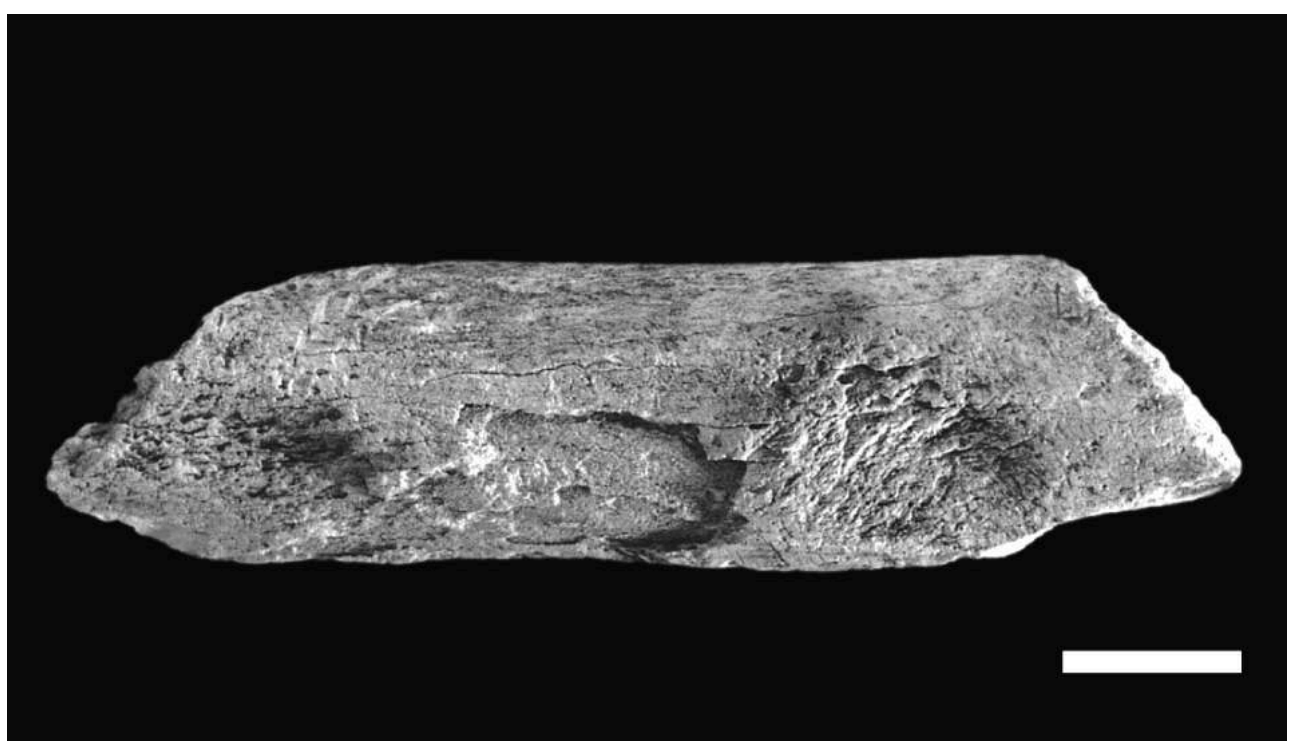

Sur les vingt retouchoirs étudiés, quatorze semblent complets. Six présentent plusieurs plages d'utilisations, dont deux avec trois plages. Les dimensions les plus récurrentes sont comprises entre 90 et $160 \mathrm{~mm}$ de longueur pour une largeur variant de 28 à $53 \mathrm{~mm}$ (fig. 9). Dans le sens longitudinal, tous les fragments sont droits ou légèrement convexes et ils sont convexes transversalement (plano-convexes).

Figure 9 - La Ferrassie - Dimensions des retouchoirs aurignaciens, sur os et sur dents (Fouilles Peyrony ; échantillon non exhaustif). Il est possible que le plus petit fragment considéré comme entier (bords de fractures lisses peu anguleux et d'angle oblique) soit en fait brisé après utilisation. Les deux fragments considérés comme incomplets mais dépassant $90 \mathrm{~mm}$ sont vraisemblablement presque complets.

Figure 9 - La Ferrassie - Dimensions of the Aurignacian retouchers on bone and teeth (Peyrony's excavations, incomplete sample). It is possible that the smallest fragment considered to be whole (smooth fracture surfaces with oblique angles) was in fact broken after utilization. The two fragments considered as incomplete but exceeding $90 \mathrm{~mm}$ in length are likely almost complete.

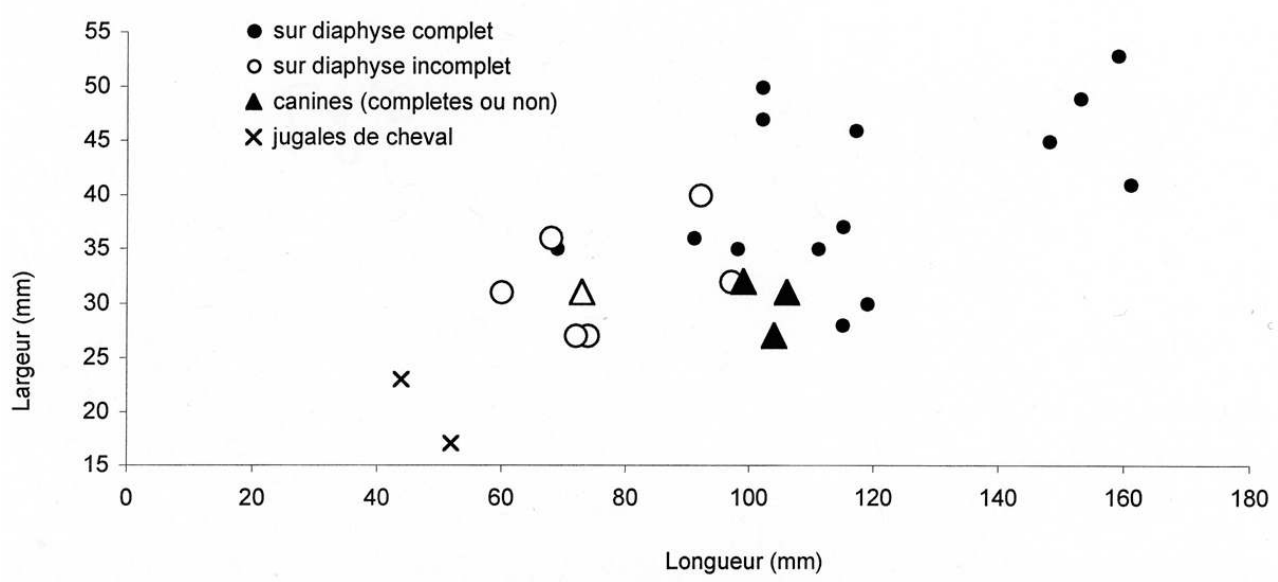

Le raclage préliminaire, caractérisé par des stries très nombreuses et parallèles, n'apparaît que sur trois supports (mais ces derniers sont très érodés). Les petites stries 
obliques très fines (stries secondaires), qui bordent parfois les plages utilisées, ne sont présentes que sur six supports. A Isturitz, C. Schwab a remarqué que ces marques sont absentes des séries moustériennes et fréquentes pour les séries du Paléolithique supérieur (Schwab 2002). A La Ferrassie, cette faible fréquence est en partie le résultat de difficultés d'identification dues à l'état de conservation assez médiocre des ossements (usure post-fouille).

Les entailles sont le plus souvent longitudinales (parallèles) ou, au contraire, perpendiculaires à l'allongement des fragments, mais des stigmates obliques sont également observables (fig. 10). Lorsque les supports sont disposés verticalement avec la plage percutée vers le haut, celle-ci est beaucoup plus décalée vers la droite que vers la gauche (fig. 11). La majorité de ces objets est donc latéralisée, comme cela a été observé dans de nombreux sites d'Europe occidentale (Taute 1965). La distance entre le centre de la plage et le bord distal ne varie pas en fonction de la longueur du fragment ; la distance moyenne est de $28 \mathrm{~mm}$ (fig. 12), ce qui semble plus important que ce qui est observé dans d'autres sites (Auguste 2002 ; Castel 1999, 2003 ; Castel et al. 1998 ; Schwab 2002, 2003). Les plages affectent généralement une forme en ellipse dont le grand axe est parallèle à l'allongement de l'objet (fig. 13). La largeur du support au niveau de la zone percutée, ou à son opposé, est très variable (fig. 14). Cette largeur n'a donc pas d'influence dans le choix de l'orientation du support. L'allongement et la robustesse de ces fragments entrent dans la variabilité des séries déjà décrites (fig. 15) mais rappelons que l'échantillon est biaisé.

Figure 10 - La Ferrassie - Retouchoirs sur diaphyses. Orientation des plages de stigmates par rapport à l'allongement de la pièce $\left(0^{\circ}\right.$ : stigmates parallèles à l'allongement du support ; $90^{\circ}$ : stigmates transversales).

Figure 10 - La Ferrassie - Retouchers on diaphyses. Orientation of the zones of use traces relative to the long axis of the piece $\left(0^{\circ}\right.$ : traces parallel to the long axis; $90^{\circ}$ : transverse traces).

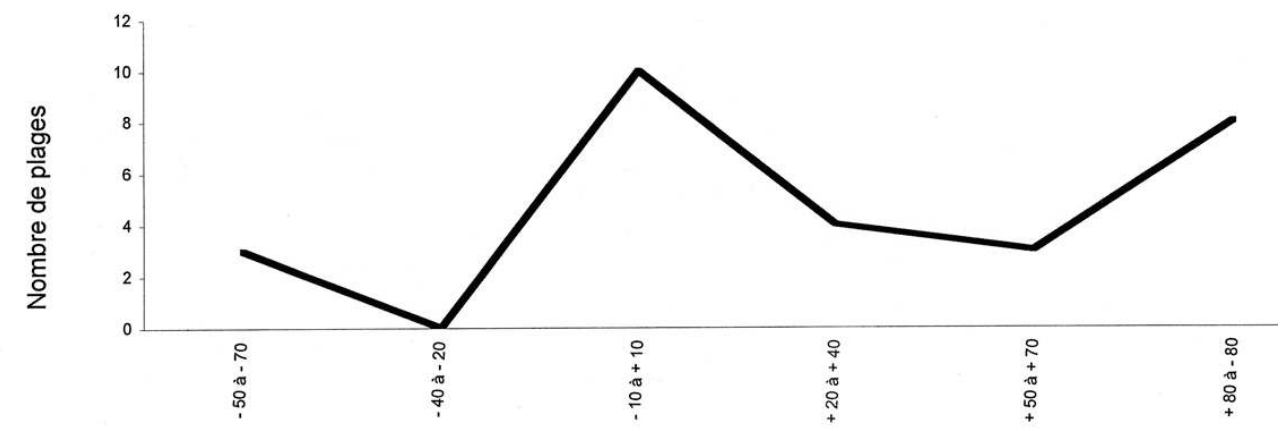

Angle (degré) 
Figure 11 - La Ferrassie - Retouchoirs sur diaphyses. Latéralisation des plages de stigmates situées près des extrémités des fragments. Dbg-Dbd : distance du bord de la plage au bord gauche - même distance... à droite. Ig-ld : distance du centre de la plage au bord gauche - même distance... à droite. Les plages de stigmates sont beaucoup plus fréquemment situées à droite lorsque le fragment est disposé verticalement.

Figure 11 - La Ferrassie - Retouchers on diaphyses. Lateralization of the zones of use traces located near the fragment extremities. Dbg-Dbd: distance of the edge of the zone on the left edge - same distance... right. Ig-Id: distance from the center of the zone on the left edge - same distance... right. The zones of use traces are more often located to the right when the fragment is vertically oriented.

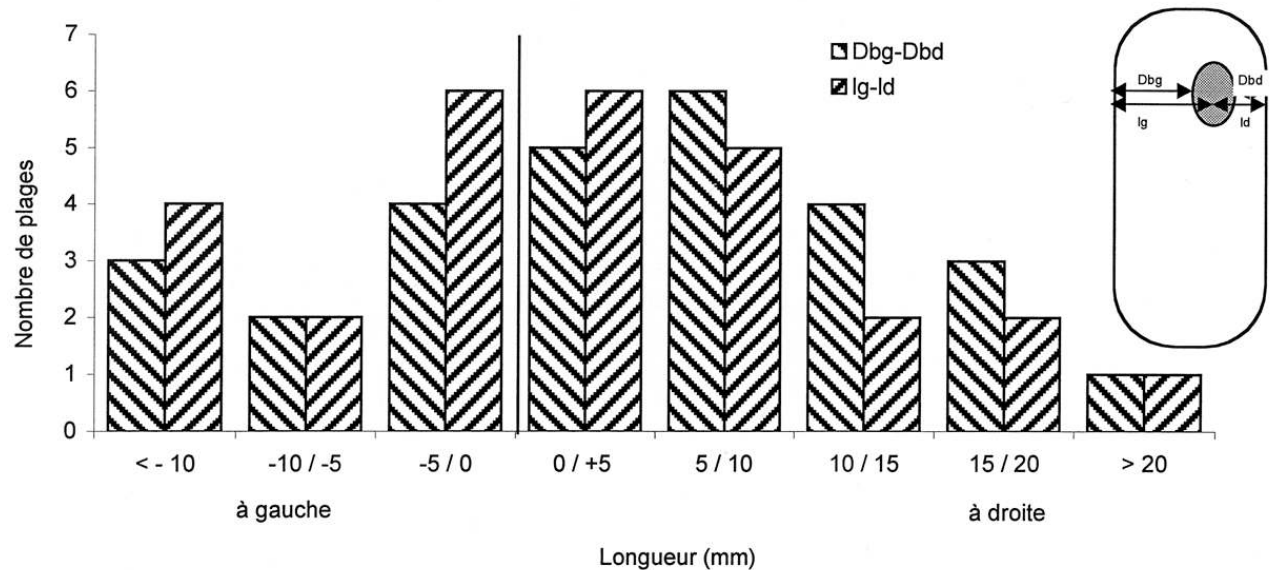

Figure 12 - La Ferrassie - Retouchoirs sur diaphyses. Localisation de la plage d'utilisation par rapport au bord distal du support.

Figure 12 - La Ferrassie - Retouchers on diaphyses. Location of the utilized zone relative to the distal edge of the object.

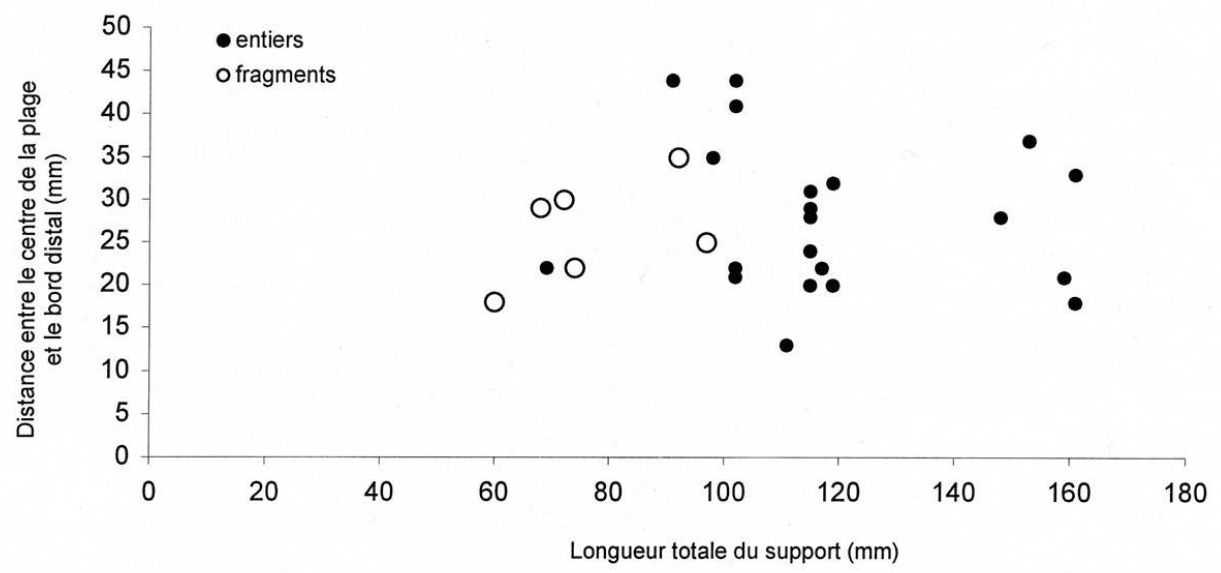


Figure 13 - La Ferrassie - Retouchoirs sur diaphyses. Dimensions des plages de stigmates. La longueur est prise dans le sens de la longueur du support (pour des angles faibles).

Figure 13 - La Ferrassie - Retouchers on diaphyses. Dimensions of the zones of use traces. The length is recorded in the long axis of the object (for weak angles).

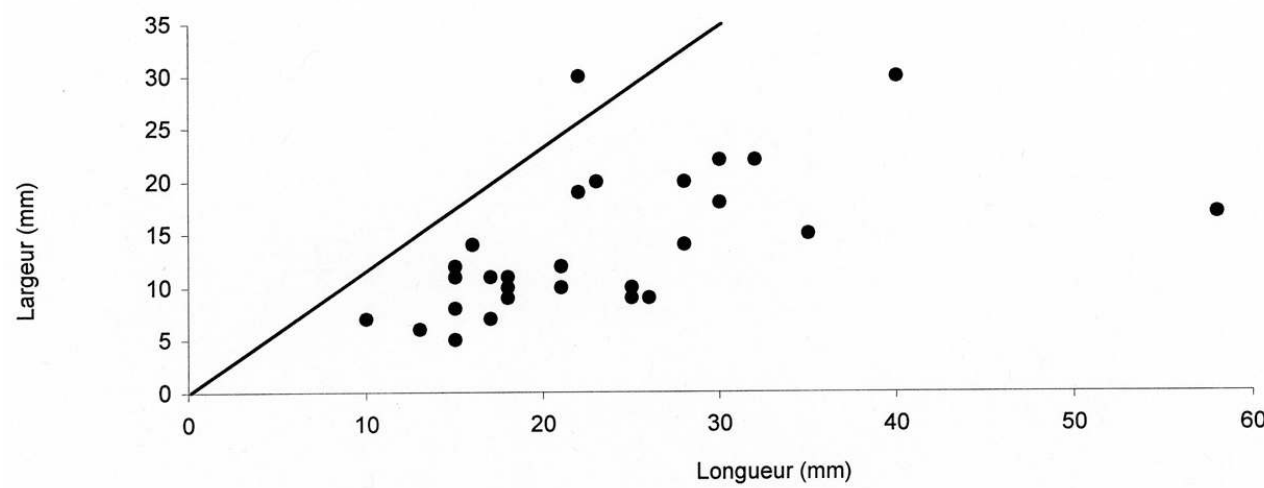

Figure 14 - La Ferrassie - Retouchoirs sur diaphyses. « Prise en main » : largeur du support au niveau de la plage d'utilisation et largeur opposée.

Figure 14 - La Ferrassie - Retouchers on diaphyses. "grip": width of the object at the utilized zone and at the opposite end.

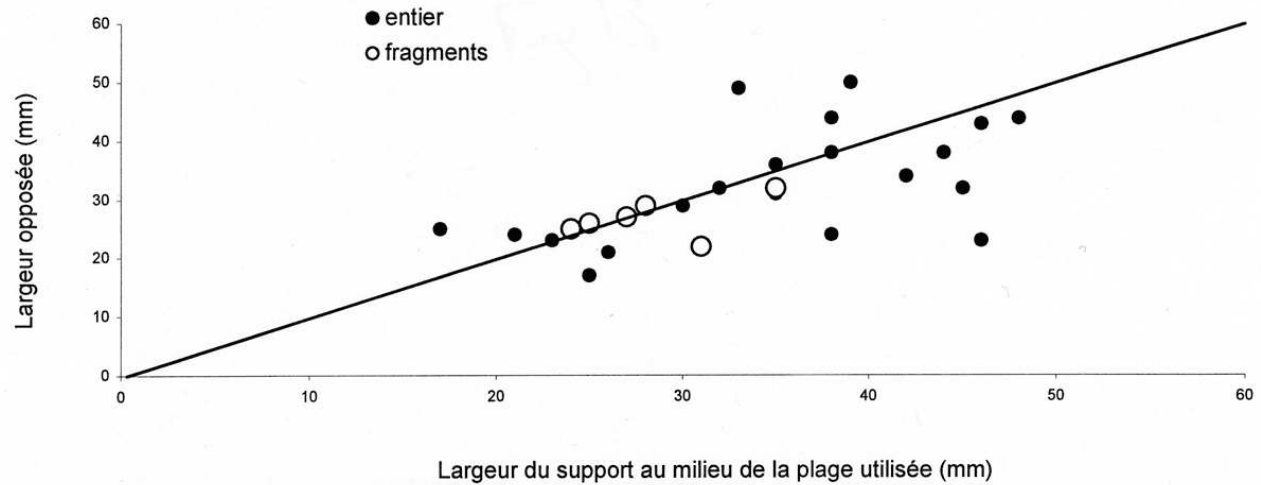

Figure 15 - La Ferrassie - Retouchoirs sur diaphyses. Morphologie générale des supports. Figure 15 - La Ferrassie - Retouchers on diaphyses. General morphology of the objects.

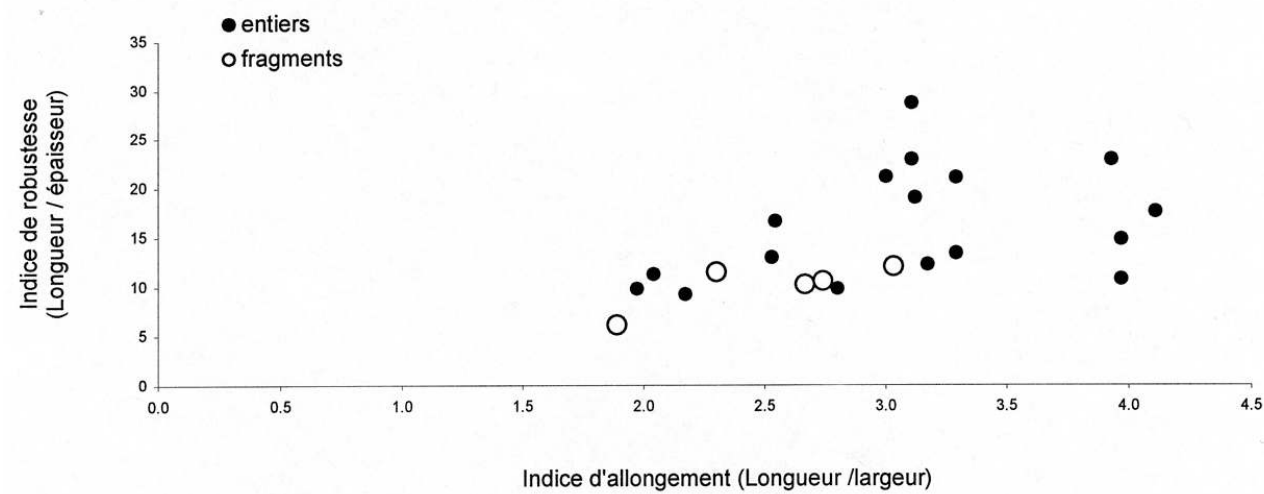

Dans l'Aurignacien de La Ferrassie, les différents paramètres mesurés indiquent que les supports choisis et le mode d'implantation des zones utilisées sont assez semblables à ceux d'autres sites paléolithiques qui ont fait l'objet de descriptions approfondies (Armand et Delagnes 1998 ; Auguste 2002 ; Castel 1999, 2003 ; Malerba et Giacobini 1998, 
2002 ; Schwab 2002 ; Taute 1965). Toutefois, la petite taille de l'échantillon et le tri dont il résulte n'autorisent guère l'interprétation des différences minimes qui existent entre La Ferrassie et les séries précédemment citées. Il en est de même pour l'intensité des marques observées et les dimensions des plages.

\section{4- Discussion}

\section{1- Caractéristiques des matériaux et hypothèses de fonctionnement} et pointue ainsi qu'une racine longue et robuste où la cavité pulpaire n'occupe qu'un mince espace à l'intérieur de la dentine (69\% de minéral). Celle-ci a un haut degré d'élasticité comparable à celui de l'os qui reste toutefois moindre que celui du bois de cervidé. Proche de l'os ( $46 \%$ de minéral), le cément ne présente pas d'organisation lamellaire. Enfin, l'émail ( $96 \%$ de minéral), comme l'os, possède une dureté considérable et une grande résistance à l'usure mais présente en revanche une relative fragilité aux chocs (Provenzano 2001). Dents et diaphyses sont donc impropres au débitage (Liolios 1992, 1999) mais restent largement utilisables dans une chaine opératoire de façonnage (retouche). D'ailleurs, nous n'avons pas découvert de fragment de matière lithique piégé sur ces retouchoirs, comme cela peut être le cas sur les percuteurs employés lors du débitage (Bordes 1974 ; Averbouh 1999). De deux choses l'une : soit les matériaux tels que l'os et les dents n'enregistrent pas les impacts comme le bois de Renne, soit il s'agit d'un autre mode de fonctionnement justement impropre à cet enregistrement. Quoi qu'il en soit, il faut noter la similarité entre les racines des canines et les diaphyses osseuses qui présenteraient finalement les mêmes caractéristiques physiques et mécaniques (Morel 1999).

Deux questions se posent alors au niveau de la distinction entre les différents types de stigmates observés sur les dents de La Ferrassie. Cette distinction renvoie-t-elle nécessairement à un même mode de fonctionnement ou à des fonctionnements différents ? Peut-elle être le reflet de plusieurs gestuelles techniques ? L'hypothèse d'un fonctionnement en percussion lancée, sur une matière d'œuvre de type solide de densité élevée (matière lithique), est retenue par la plupart des auteurs qui ont travaillé sur le sujet. Toutefois, comme le suggèrent les incisions très localisées dans l'usure naturelle de la couronne de la canine B 435.5 ou la molaire supérieure de Cheval, ne peut-on pas envisager, comme Semenov (1964) et Shchelinskii (1983; voir notamment Shchelinskii in Plisson, 1988), la pression comme méthode propice à la formation des stigmates observés?

\section{2- Mise en place d'un protocole expérimental}

Si l'origine des stigmates observables sur les retouchoirs en os a déjà pu faire l'objet d'hypothèses étayées par un référent expérimental (Rigaud 1977, par exemple), il n'en est pas de même pour les canines de grands carnivores ou les molaires de Cheval. Par conséquent, afin d'essayer d'interpréter, en termes de gestuelle technique, les stigmates observés sur le matériel de La Ferrassie, nous avons élaboré un protocole expérimental qui met en œuvre une matière dure animale (pour des raisons pratiques, 
c'est l'ivoire d'hippopotame qui a été utilisé) ainsi qu'une matière lithique (silex) (tabl. 5).

Tableau 5 - Protocole d'utilisation des retouchoirs expérimentaux sur ivoire. Table 5 - Procedure for the experimental use of ivory retouchers.

\begin{tabular}{|c|c|c|c|}
\hline & Expérience 1 & Expérience 2 & Expérience 3 \\
\hline Matériau de l'outil - retouchoir & \multicolumn{2}{|c|}{ ivoire } & ivoire \\
\hline Poids (en grammes) & \multicolumn{2}{|c|}{62,7} & 33,7 \\
\hline Morphologie partie active du retouchoir & \multicolumn{2}{|c|}{ convexe } & convexe \\
\hline Objet et matière percuté & \multicolumn{2}{|c|}{ lames de silex } & lames de silex \\
\hline Nombre d'objets percutés & \multicolumn{2}{|c|}{10} & 4 \\
\hline Type de percussion & \multicolumn{2}{|c|}{ percussion lancée } & pression \\
\hline Inclinaison des lames & $0-45^{\circ}$ & $45-90^{\circ}$ & $45^{\circ}$ \\
\hline $\begin{array}{l}\text { Gestuelle } \\
\text { Gynérimentateur }\end{array}$ & \multirow{2}{*}{\multicolumn{2}{|c|}{$\begin{array}{l}\text { polyphasée } \\
\text { droitier }\end{array}$}} & monophasée \\
\hline Experimentateur & 0 & & aroctier \\
\hline $\begin{array}{l}\text { Type stigmate sur retouchoir } \\
\text { Type retouche sur lames }\end{array}$ & semi-abru & $\begin{array}{l}2,3 \\
\text { à abruptes }\end{array}$ & $\begin{array}{c}\text { proches du type } 2 \\
\text { abruptes }\end{array}$ \\
\hline
\end{tabular}

37 Trois pièces en ivoire ont été utilisées comme retouchoirs. Il s'agit de trois volumes allongés, épais et de section plano-convexe. Les deux premières pièces ont été utilisées de manière ininterrompue durant 30 minutes puis à nouveau employées 1 mois plus tard dans les mêmes conditions (fig. 16.1 et 2). Un troisième retouchoir a servi durant 30 minutes sans être réutilisé par la suite (fig. 16.3).

Figure 16 - Retouchoirs expérimentaux en ivoire employés pour façonner des lames en silex. Les pièces 1 et 2 ont été utilisées en percussion lancée. La pièce $n^{\circ} 3$ a servi à la transformation de lames par retouche à la pression. Les stigmates engendrés sont proches des stigmates de type 1 et 2 observés sur les dents archéologiques de La Ferrassie.

Figure 16 - Experimental ivory retouchers used for the "façonnage" of flint blades. Tools 1 and 2 were used for percussion. The number 3 was used to transform flint blades by pressure flaking. Visible traces seem like the type 1 and 2 traces observed on the archaeological teeth of La Ferrassie.

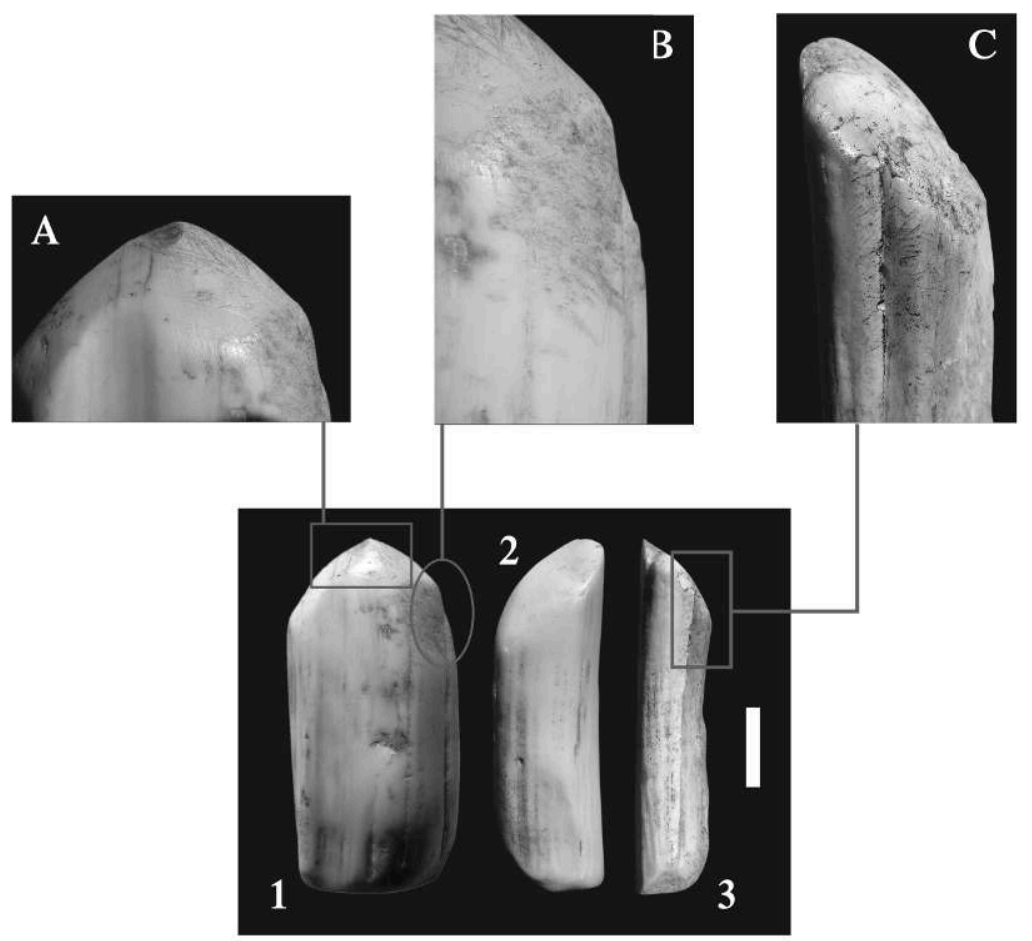


Deux objets en ivoire, tenus fermement dans la main droite, ont été utilisés en percussion lancée (expérimentateur: F.-X. Chauvière) pour façonner, par retouche directe, des lames de silex débitées au percuteur tendre (tailleur: M. Brenet). Nous avons fait varier l'inclinaison des lames, tenues dans la main gauche $\left(0-45^{\circ}\right.$ et $\left.45-90^{\circ}\right)$, sans changer la trajectoire de percussion du retouchoir qui est restée linéaire. L'angle d'incidence entre la pièce en ivoire et la lame de silex, au point de contact, est donc soit de $45^{\circ}$, soit proche de $90^{\circ}$.

La retouche par pression a également été expérimentée pour façonner, par retouche directe et inverse, des lames de silex à l'aide d'un retouchoir en ivoire. L'angle d'incidence, au point de contact, entre le retouchoir et la pièce lithique a toujours été proche de $45^{\circ}$.

\section{3- Quelles gestuelles techniques pour quels stigmates?}

\section{Percussion lancée ou pression?}

Sur les lames de silex, la percussion lancée produit des retouches semi-abruptes à abruptes, proches de la retouche aurignacienne. Sur les retouchoirs en ivoire, elle engendre des stigmates de type 1 et 2 (fig. 16.1-2). Les stigmates de type 1 renvoient à un angle d'incidence de $45^{\circ}$ entre la lame et le retouchoir alors que les stigmates de type 2 renvoient à un angle d'incidence de $90^{\circ}$.

41 La pression produit, sur les lames de silex, des retouches abruptes. Les stigmates observés sur le retouchoir en ivoire sont proches de ceux du type 2 mais les stries sont beaucoup plus courtes (fig. 16.3) et ne sont donc pas vraiment équivalentes à ce que l'on observe sur la canine B.435.3, mais se rapprochent de ce qui a été distingué dans le cas de la molaire supérieure de Cheval.

\section{L'apport de l'expérimentation : que conclure?}

La démarche expérimentale présentée ici revêt encore un caractère largement exploratoire. Elle doit être pondérée par le fait que d'une part, un seul expérimentateur (droitier) a été mis à contribution et d'autre part, celui-ci n'est qu'un artisan occasionnel du travail du silex.

Plusieurs observations nous semblent toutefois importantes à signaler :

- [50 sur les lames de silex, les retouches effectuées par percussion lancée ou par pression peuvent trouver des équivalents dans le matériel archéologique aurignacien de La Ferrassie (lames retouchées, troncatures, lames étranglées). Il est donc possible d'envisager une relation fonctionnelle entre canines et molaires de Cheval et industrie lithique ;

- [50 sur les retouchoirs expérimentaux, les stigmates produits par percussion lancée ou par retouche peuvent être distingués si l'on considère leur longueur respective et leur morphologie ;

- [⿷匚⿱⿰㇒一大口日 la formation de stigmates de morphologies différentes peut trouver une origine dans une gestuelle différente ;

- [50 si la cinématique d'un retouchoir reste la même, que l'utilisation soit ininterrompue ou interrompue et effectuée par un même expérimentateur, les stigmates sont toujours localisés aux mêmes endroits, ce qui contribue à la formation des coalescences de stries observées sur le matériel archéologique. A ce titre, la localisation des stigmates sur la 
quasi-totalité des volumes des canines permet de poser l'hypothèse d'une utilisation polyphasée de ces dents (interruption dans la gestuelle technique, réutilisation de l'objet par d'autres individus), à l'inverse des retouchoirs sur diaphyses osseuses dont les stigmates de travail forment des concentrations bien définies. Une telle hypothèse n'est pas incompatible avec ce que l'on connaît, en terme tracéologique, des usures des perforations des canines de grands fauves utilisées comme objets de parure, qui témoignent d'un temps d'utilisation prolongée. Par conséquent, ceci nous amène à penser que les retouchoirs sur canines de grands fauves sont des objets symboliques ou à « valeur ajoutée » que l'on conserve sur soi et que l'on véhicule dans le temps et l'espace, en raison de leur rareté, de la difficulté à se les procurer. En revanche, les retouchoirs sur diaphyses osseuses, issus de la chaîne alimentaire, constituent des matériaux plus facilement accessibles. En général, les lieux d'acquisition et d'abandon coïncident avec l'espace d'utilisation mais ce n'est pas toujours le cas (Castel 1999, 2003).

\section{5- Bilan et perspectives de recherche} dans de nombreux gisements aurignaciens ou dans d'autres sites du Paléolithique supérieur, la nature anatomique des éléments n'est généralement pas détaillée. Dans certains cas, leur présence est associée à des activités de carnivores. Mais la plupart du temps, ils constituent les indices isolés d'une acquisition par l'homme, elle-même dissociée de la chaîne alimentaire, dont il ne faut pas tenir compte pour caractériser l'environnement exploité. Les canines de grands carnivores sont en effet des pièces dont la rareté, soumise à une variation plus ou moins forte de la disponibilité d'Ursus spelaeus et de Panthera dans le temps et l'espace (Fosse et al., 2002), accentue, peut-être, le fort pouvoir symbolique. Il n'est pas certain que ces animaux aient été chassés dans un environnement proche des sites et la récupération de ces dents sur des carcasses d'animaux morts est probable 6 . Mais, à notre sens, seule une approche de type archéozoologique des restes de grands fauves, telle celle développée pour Ursus spelaeus (Auguste 2003; Fosse et al. 2001, 2002) peut, à terme, permettre de sérier les observations techniques.

L'analyse technique et fonctionnelle des retouchoirs sur dents et sur os compact de La Ferrassie conduit en premier lieu à conclure à une certaine similarité entre ces deux matériaux, tant au niveau de leurs caractéristiques mécaniques qu'en ce qui concerne les surfaces convexes utilisées. La diversité des plages d'utilisation sur les canines indique également qu'il pourrait s'agir d'outils à tout faire, en tout cas plus polyvalents que les retouchoirs sur diaphyses dont les potentialités sont limitées par les caractéristiques mécaniques et morphométriques de l'os.

Ce type d'approche permet également d'introduire, à l'échelle des objets eux-mêmes, des césures temporelles dans leur utilisation. Cette mise en évidence d'une gestuelle mono- ou polyphasée, selon les volumes et les matériaux, permet d'argumenter la gestion différentielle des objets techniques dans le temps mais aussi dans l'espace. Elle permet aussi de distinguer des objets de "l'instant", à durée d'utilisation courte et contingente comme les retouchoirs sur os (Armand et Delagnes 1998 ; Castel 1999, 2003) et sur dents de Cheval, et des objets du "temps long ", largement véhiculés et employés que sont les retouchoirs sur canines de grands fauves. Si cette idée de gestuelle 
polyphasée n'avait guère été proposée par les différents auteurs qui ont travaillé sur les retouchoirs 7 , en revanche, des considérations similaires ont été exposées à propos d'autres catégories d'objets, notamment celles considérées comme des «systèmes artificiels à mémoire » (D’Errico 1998).

Les dents jugales de Cheval qui portent des traces d'utilisation, bien que présentes dans le Moustérien et l'Aurignacien (c'est-à-dire deux groupes culturels bien distincts), sont rares mais il s'agit en fait d'une catégorie de vestiges encore peu connue. Elles n'ont vraisemblablement pas de fonction symbolique et leur usage est beaucoup plus opportuniste 8. Si ces vestiges se révélaient spécifiques du Moustérien et de l'Aurignacien, comme cela semble le cas dans l'état actuel des recherches, il serait nécessaire d'identifier quels comportements techniques (ou symboliques?) communs pourraient les lier. Leur absence des phases plus récentes du Paléolithique supérieur peut surprendre compte tenu de la grande continuité observée parmi les retouchoirs sur diaphyses de l'Aurignacien au Magdalénien.

A cette mise en relation, dans un cadre technique et culturel, de ces vestiges différents qui ont fonctionné de manière similaire doivent se joindre les équivalents lithiques aurignaciens de ces outillages (galets à grain fin utilisés sur leurs faces planes), en tentant notamment de déterminer si les caractéristiques d'utilisation relèvent d'un même cadre de fonctionnement et/ou d'usage. Au préalable, un inventaire plus précis de ces catégories de vestiges à travers le Paléolithique moyen et le Paléolithique supérieur s'avère indispensable afin de déterminer la portée de nos conclusions réalisées à partir du matériel très particulier de La Ferrassie.

\section{Remerciements}

Nous adressons nos plus vifs remerciements aux personnes suivantes qui nous ont apporté leur précieuse contribution pour la rédaction de ce manuscrit:

Jean-Jacques Cleyet-Merle, conservateur, André Morala, assistant-ingénieur, Philippe Jugie, photographe, et l'ensemble du personnel du Musée National de Préhistoire qui a facilité notre recherche;

Florence Cerceau, infographiste du Muséum d'histoire naturelle de Genève ; Nathalie Mémoire, directrice du Muséum d'histoire naturelle de Bordeaux, Sigolène Loizeau conservatrice au Musée d'Aquitaine, René Castanet du Musée archéologique de Sergeac pour les informations concernant les collections dont ils ont la charge;

Dominique Armand, pour sa contribution aux réflexions sur les retouchoirs atypiques, Jean-Guillaume Bordes, Randall White, Jean-Pierre Chadelle et Christophe Griggo, pour leurs informations sur les sites paléolithiques du Sud-ouest de la France;

Louis Chaix, professeur à l'Université de Genève, qui nous a fait part de ses connaissances sur les grands carnivores;

Patrick Bidart, Directeur d'Ethnicité, pour ses conseils judicieux sur l'industrie osseuse ;

Michel Brenet, INRAP Grand Ouest, pour nous avoir fourni les lames de silex nécessaires à

l'expérimentation;

\section{Crédits photographiques}

Philippe Jugie du Musée National de Préhistoire sauf: fig. 7.2 et 7.3 et fig. 16 : Jean-Christophe Castel. Travail des photos et mise en forme par Florence Marteau du Muséum d'histoire naturelle de Genève. 


\section{BIBLIOGRAPHIE}

ARMAND D. et DELAGNES A. 1998 - Les retouchoirs en os d'Artenac (couche 6c) : perspectives archéozoologiques. In : J.-Ph. Brugal, L. Meignen, M. Patou-Mathis (Ed.), Economie préhistorique : les comportements de subsistance au Paléolithique, XVe Rencontres internationales d'archéologie et d'Histoire d'Antibes, Editions APDCA, Sophia Antipolis, 1998, p. 205-214, 8 fig., 1 tabl.

AUGUSTE P. 2002 - Fiche éclats diaphysaires du Paléolithique moyen : Biache-saint-Vaast (Pas-deCalais) et Kulna (Moravie, République Tchèque), In : M. Patou-Mathis (Dir.), Compresseurs, percuteurs, retouchoirs ... Os à impressions et éraillures, Industrie de l'os préhistorique, cahier X, Paris, Editions de la Société Préhistorique Française, 2002, p. 39-57, 7 fig., 15 tabl.

AUGUSTE P. 2003 - La chasse à l'ours au Paléolithique moyen : mythes, réalités et état de la question, In : M. Patou-Mathis, H. Bocherens (Eds), Le rôle de l'environnement dans les comportements des chasseurs-cueilleurs préhistoriques. BAR International Series, 1105, p. 135-142.

AVERBOUH A. 1999 - Un fragment de percuteur sur partie basilaire du site magdalénien d'Enlène (Ariège), Bulletin de la Société préhistorique Française, 1999, tome 96, nº 4, p. 497-504, 3 fig.

BALLESIO R. 1975 - Etude de Panthera (Leo) spelaea (Goldfuss) nov. sub. sp. (Mammalia, Carnivora, Felidae) du gisement pléistocène moyen des Abîmes de la Fage à Noailles (Corrèze). Nouvelles Archives du Muséum d'Histoire Naturelle de Lyon, fasc. 13, p. 47-55, 5 tabl..

BAUDOIN M., CHAUVET G., HENRI-MARTIN L., HUE E. et TATE 1907 - Discussion sur l'usage de l'os comme outil à l'époque moustérienne. , Bulletin de la Société Préhistorique de France, 1906, t. 4, p. $189-200$.

BEGOUEN H. et BREUIL H. 1958 - Les cavernes du Volp. Trois Frères - Tuc d'Audoubert à MontesquieuAvantès (Ariège), Paris, Arts et métiers graphiques, 1958, 121 p., 115 fig.

BEGOUEN R. et CLOTTES J. 1982 - Des ex-votos magdaléniens ? La Recherche, avril 1982, nº 132, vol. 13, p. 518-519., 2 fig.

BON F. 2002 - L'Aurignacien entre Mer et Océan. Réflexion sur l'unité des phases anciennes de l'Aurignacien dans le sud de la France, Mémoire XXIX de la Société Préhistorique Française, 2002, 253 p., 81 fig., 43 pl.

BONIFAY M.-F. 1971 - Carnivores quaternaires du Sud-Est de la France. Mémoires du Muséum National d'Histoire Naturelle, Nouvelle série. T. XXI, fasc. 2. Paris, Editions du Museum. 377 p.,76 fig., 109 tabl.,27 pl. h.t.

BORDES F. 1974 - Percuteur en bois de renne du Solutréen supérieur de Laugerie-Haute Ouest, Premier colloque international sur l'industrie de l'os dans la Préhistoire, Abbaye de Sénanque, 1974, p. 97-100.

BOSINSKI G. 1990 - Homo Sapiens. L'histoire des chasseurs du Paléolithique sup. en Europe (40000-10000 av. J.-C.), Errance, 1990, 281 p., ill.

BROOKS A. S. 1995 - L'Aurignacien de l'abri Pataud, niveaux 6 à 14, In : H. M. BRICKER (Dir.), Le Paléolithique supérieur de l'abri Pataud (Dordogne) : les fouilles de H.L. Movius Jr., Paris, Maison des Sciences de l'Homme, Documents d'Archéologie Française, n 50, p. 167-225, 25 fig., 15 tabl.

CAPITAN L. et PEYRONY D. 1912 - Station préhistorique de La Ferrassie, Commune de Savignacdu-Bugue (Dordogne), Revue anthropologique, $n^{\circ} 2$, p. 76-99, 35 fig. 
CASTEL J.-C. 1999 - Comportements de subsistance au Solutreén et au Badegoulien d'après les faunes de Combe-Saunière (Dordogne) et du Cuzoul de Vers (Lot). Thèse, Université Bordeaux I, non publiée, 619 p., ill.

CASTEL J.-C. 2003 - Économie de chasse et d'exploitation de l'animal au Cuzoul de Vers (Lot) au Solutréen et Badegoulien, Bulletin de la Société préhistorique Française, tome 100, 1, p. 41-66, 11 fig., 12 tabl.

CASTEL J.-C., LIOLIOS D., CHADELLE J-P. et GENESTE J-M. 1998 - De l'alimentaire et du technique : une chaîne opératoire de consommation du renne dans le Solutréen de Combe-Saunière. In :J.-P. Brugal, L. Meignen, M. Patou-Mathis (Ed.), Economie préhistorique : les comportements de subsistance au Paléolithique, XVe Rencontres internationales d'archéologie et d'Histoire d'Antibes, Editions APDCA, Sophia Antipolis, 1998, 434-450, 8 fig.

CHAMPAGNE F. et ESPITALIE R. 1981 - Le Piage, site préhistorique du Lot. Mémoire XV de la Société Préhistorique Française, 1981, 205 p, ill.

CHAUVIERE F.-X. 1997 - Les dents travaillées de Combe-Saunière : approche économique et fonctionnelle, rapport d'étude, $24 \mathrm{p}$.

CHAUVIERE F.-X. 2001 - La collection Chaplain-Duparc des musées du Mans : nouveaux éléments d'interprétation pour la “ sépulture Sorde 1 ” de Duruthy (Sorde l'Abbaye, Landes), Paléo, 13, p. 89-110, 18 fig., 5 tabl., 1 graph.

CHEYNIER A. 1963 - La caverne de Pair-Non-Pair, Gironde, Fouilles de François Daleau, Documents d'Aquitaine III, Publications de la Société archéologique de Bordeaux, 1963, 213 p., 60 fig., 7 pl.

CLOT A. 1982 - La Grotte de la Carrière (Gerde, Haute-Pyrénées). Stratigraphie et paléontologie des carnivores. Thèse, Université Paul Sabatier de Toulouse. Non publiée.

CLOTTES J. (Dir.) 2001 - La grotte Chauvet. L'art des origines, Coll. Arts rupestres, Le Seuil, 2001, 225 p., 206 fig.

DELPECH F. 1983 - Les faunes du Paléolithique Supérieur dans le Sud-Ouest de la France, Cahiers du Quaternaire, $\mathrm{n}^{\circ}$ 6, 456 p., ill.

DELPECH F. 1984 - La Ferrassie : Carnivores, Artiodactyles et Périssodactyles. In : H. Delporte (Dir.), Le grand abri de La Ferrassie : fouilles 1968-1975. p. 61-89. Etudes Quaternaires, mém. nº 7., p. 61-90, IV pl., tabl.

D'ERRICO F. 1998 - Des encoches aux ordinateurs : l'origine des moyens artificiels de stockage de l'information, In : A. Ducros, J. Ducros, F. Joulian (Dir.), La culture est-elle naturelle? Histoire, épistémologie et applications récentes du concept de culture, Paris, Editions Errance, Collections des Hespérides, p. 199-216, 6 fig.

FAVRAUD A. 1907 - Station aurignacienne au Pont-Neuf, commune de la Couronne (Charente), Revue de l'Ecole d'Anthropologie de Paris, t. XII, p. 418-428, 7 fig.

FOSSE Ph., en collaboration avec JAUZION G., MAKSUD F., QUETTIER D., QUETTIER R., ROUCH Ph. et BESSON J.-P. 2001 - Ursidés pléistocènes des Pyrénées : éléments de paléontologie et de paléobiologie, Bulletin de la Société Préhistorique Ariège-Pyrénées, t. LVI, 2001, p. 103-138, 8 fig., 13 tabl.

FOSSE Ph., MOREL Ph. et BRUGAL J. Ph. 2002 - Taphonomie et paléoethologie des Ursidés pléistocènes. In : Th. Tillet et L.R. Binford (Dir.) : L'ours et l'Homme, Actes du Colloque d'Auberivesen-Royans (Isère), 1997, Liège, ERAUL n 100, p. 79-101, 12 fig., 9 tabl. 
HAHN J. 1977 - Aurignacien. Das äaltere Jungpaläolithikum in Mittel-und Osteuropa, Köln, Wien, Fundamenta, Bölhau Verlag, n 9, 355 p., 19 tabl., 187 pl., 5 cartes.

HENRI-MARTIN L. 1907-1910 - Recherches sur l'évolution du Moustérien dans le gisement de la Quina. I Industrie osseuse, Paris, Schleicher, 316 p., 67 pl.

HENRI-MARTIN L. 1908 - note à la suite de la communication de A. Rutot : Sur la répartition des os utilisés en Belgique, Bulletin de la Société Préhistorique de France, 1908, tome 11, p. 281.

KOBY F.E. 1952 - La dentition lactéale d'Ursus spelaeus. Revue Suisse de Zoologie, t. 59, n²7, p. 511-541, 17 fig.

LEROI-GOURHAN A. 1943 - Evolution et techniques. L'homme et la matière, Paris : Editions Albin Michel, coll. Sciences d'aujourd'hui. 367 p., 577 fig.

LEROY-PROST Ch. 1975 - L'industrie osseuse aurignacienne. Essai régional de classification : Poitou, Charente, Périgord. Gallia Préhistoire, t. 18, fasc. 1, p. 65-156, 27 fig.

LEROY-PROST Ch. 1979 - L'industrie osseuse aurignacienne. Essai régional de classification : Poitou, Charente, Périgord. Gallia Préhistoire, t. 22, fasc. 1, p., 205-370, 100 fig.

LEROY-PROST Ch. 1996 - Les “cousoirs” aurignaciens sur canines de carnivores, Antiquités Nationales, t. 28, p. 47-52

LEROY-PROST Ch. 2002 - Fiches canines de carnivores, In : M Patou-Mathis (Dir.), Compresseurs, percuteurs, retouchoirs ... Os à impressions et éraillures, Industrie de l'os préhistorique, cahier $\mathrm{X}$, Paris, Editions de la Société Préhistorique Française, p. 99-104, 4 fig.

LIOLIOS D. 1992 - Approche des relations techno-économiques entre le lithique et l'os dans l'Aurignacien d'Aquitaine, Mémoire de maîtrise en Ethnologie et Préhistoire, Université de Paris X-Nanterre, 154 fig., 13 fig. Non publié.

LIOLIOS D. 1999 - Variabilité et caractéristiques du travail des matières osseuses au début de l'Aurignacien : approche technologique et économique, Thèse de doctorat en Ethnologie et Préhistoire, Université de Paris X-Nanterre., 359 p., 54 fig., 59 tabl. Non publiée.

MADELAINE S. 1989 - Contribution des anciennes fouilles à la connaissance des ongulés et de leurs milieux durant le Würm récent en Dordogne. Paléo, 1, p. 36-46.

MALERBA G. et GIACOBINI G. 1998 - Les restes fauniques du paléolithique moyen de la grotte de San Bernardino (Vicenza, Italie du Nord orientale) : analyse micromorphologique des surfaces osseuses, XIIIème congrès de l'Union Internationale des sciences Préhistoriques et protohistoriques, Forli, Italie, 1996, vol. 6, t. 1, p. 264-268, 4 fig.

MALERBA G. et GIACOBINI G. 2002 - Fiche éclats diaphysaires avec marques transversales d'utilisation, In : M Patou-Mathis (Dir.), Compresseurs, percuteurs, retouchoirs ... Os à impressions et éraillures, Industrie de l'os préhistorique, cahier X, Paris, Editions de la Société Préhistorique Française, p. 29-37, 6 fig.

MOREL P. 1999 - L'Homme et l'Animal au cours des millénaires, Cours d'introduction à l'archéozoologie et à l'Anthropologie, Institut de préhistoire, Université de Neuchâtel, polycopié non publié.

MOREL Ph. et GARCIA M.-A. 2002 - La chasse à l'ours dans l'art paléolithique. In : Th. Tillet et L.R. Binford (Dir.) : L'ours et l'Homme, Actes du Colloque d'Auberives-en-Royans (Isère), 1997, Liège, ERAUL $n^{\circ} 100$, p. 219-227, 14 fig., 1 tabl. 
MOUTON P. 1957 - Un nouvel outil de l'Aurignacien typique, “ les cousoirs ” sur canines de grands fauves. Congrès préhistorique de France, Comptes rendus de la XVe session, Poitiers - Angoulême, 1956. Paris, Société Préhistorique Française, p. 756-757.

MOUTON P. et JOFFROY R. 1958 - Le gisement aurignacien des Rois à Mouthiers (Charente), Paris, CNRS, IXème suppl. à Gallia, 142 p., 46 fig.

MÜNZEL S.C., LANGGUTH K., CONARD N.J., UERPMANN H.-P. 2001 - Höhlenbärenjagd auf der schwäbischen Alb vor 30.000 Jahren. Archeologisches Korrespondenzblatt, 31, p. 317-328.

PALES L. 1969 - Les gravures de la Marche, I.- Félins et ours, Publications de l'Institut de préhistoire de l'Université de Bordeaux , mémoire 7, Bordeaux, Imprimerie Delmas, 1969, 118 p., $41 \mathrm{pl}$.

PATOU-MATHIS M. (Dir.) 2002 - Compresseurs, percuteurs, retouchoirs ... Os à impressions et éraillures, Industrie de l'os préhistorique, cahier X, Paris, Editions de la Société Préhistorique Française, 136 p., ill.

PROVENZANO N. 2001 - Produits, techniques et productions à l'âge du Bronze. L'industrie osseuse dans les Terramares de la moyenne vallée du Pô, Thèse, Université de Provence - Centre d'Aix, 2 vol.

PEYRONY D. 1933 - Les industries “ aurignaciennes ” dans le bassin de la Vézère. Bulletin de la Société Préhistorique Française, t. 30, p. 543-558.

PEYRONY D. 1934 - La Ferrassie, Préhistoire, t. 3, p. 1-92, 89 fig.

PEYRONY D. 1937 - Le Périgordien et l'Aurignacien. Nouvelles observations. Bulletin de la Société Préhistorique Française, t. 34, p. 616-619.

PLISSON H. 1988 - Technologie et tracéologie des outils lithiques moustériens en Union Soviétique : les travaux de V. E. Shchelinskiï. In : L'Homme de Néandertal, vol. 4, La technique, Liège, ERAUL $n^{\circ} 31,1988$ p. 121-168.

RIGAUD A. 1977 - Analyses typologiques et technologique des grattoirs magdaléniens de la Garenne à Saint-Marcel (Indre), Gallia Préhistoire, t. 20, fasc.1 , p. 1-43, 34 fig., 2 tabl.

ROUSSEAU M. 1967 - Les grands félins dans l'art de notre préhistoire, Paris, Ed. Picard, 200 p., 125 fig., 14 tabl., h. t.

ROUZAUD F. 1997 - L'ours dans l'art paléolithique. In : Th. Tillet et L.R. Binford (Dir.) : L'ours et l'Homme, Actes du Colloque d'Auberives-en-Royans (Isère), 1997, Liège, ERAUL n 100, p. 229-234, 2 fig.

RUTOT A., 1908 - Sur la répartition des os utilisés en Belgique, Bulletin de la Société Préhistorique de France, 1908, tome 11, p. 280-281.

SCHWAB C. 2002 - Fiche éclats diaphysaires du Paléolithique moyen et supérieur : la grotte d'Isturitz (Pyrénées-Atlantiques), In : M Patou-Mathis (Dir.), Compresseurs, percuteurs, retouchoirs ... Os à impressions et éraillures, Industrie de l'os préhistorique, cahier X, Paris, Editions de la Société Préhistorique Française, p. 59-73, 17 fig., 32 tabl..

SCHWAB C. 2003 - Les os à impressions et à éraillures de la grotte d'Isturitz (PyrénéesAtlantiques, France), In : M Patou-Mathis, P. Cattelain, D. Ramseyer (Coord.), L'industrie osseuse pré et protohistorique en Europe. Approches techniques et fonctionnelles, Actes du colloque 1.6, XIVe Congrès de l'UISPP, Liège, 2-8/09/2001, T. XXVI, 2003, p. 9-18, 13 fig.

SEMENOV S. A. 1964 - Prehistoric technology. An experimental study of the oldest tools and artefacts from traces of manufacture and wear. Cory, Adams and Mackay, London. 
SHCHELINSKII V. E. 1983 - K izoutcheniïou tehniki, tehnologii iznotovleniïa i founktiï oroudiï moust'erskoï epohou (Vers une étude de la technique, de la technologie de fabrication et de la fonction des outils de l'époque moustérienne) In: Tehnologiïa proizvodstva v epohypaleolita, Naouka, Leningrad, p. 72-133.

SIGAUT F. 1991 - Un couteau ne sert pas à couper mais en coupant. Structure et fonction dans l'analyse des objets. In : 25 ans d'études technologiques en Préhistoire, Xe rencontres internationales d'Archéologie et d'Histoire d'Antibes, Editions APDCA, Juan-les-Pins, 1991, p. 21-34, 7 fig.

SONNEVILLE-BORDES D. de 1960 - Le Paléolithique supérieur en Périgord, Bordeaux : Delmas, 2 tomes, 549 p., 294 fig., LXIV tabl.

SUIRE C., 1968 - A propos d'une canine inférieure de félin, Felis cf. pardus L. découverte à La Chaise (Charente). Actes Soc. linnéenne. Bordeaux,1968, 105,série B - N 5,5p.,4 fig.

SUIRE C. 1970 - Contribution à l'étude des dents de « Felis spelaea » Gold. Bull. del'AFEQ,1970-4,p. 243-252,6 fig., 2 tabl.

TAUTE W. von 1965 - Retoucheure aus Knochen, Zahnbein, und Stein vom Mittelpaläolithikum bis zum Neolithikum, Fundberichte aus Schwaben, t. 17, p. 76-102.

TEXIER J.-P. 2001 - Sédimentogénèse des sites préhistoriques et représentativité des datations numériques. In : J.N. Barandon, P. Guibert, V. Michel (Ed.), Datation, Actes des XXIe rencontres internationales d'Antibes, Editions APDCA, Sophia Antipolis, 2001, p. 159-175, 11 fig.

VINCENT A. 1993 - L'outillage osseux au Paléolithique moyen : une nouvelle approche. Thèse de doctorat en Ethnologie et Préhistoire, Université de Paris X-Nanterre., non publiée.

\section{NOTES}

1. L'abri du Pigeonnier (Gensac, Gironde) a été fouillé au cours des années 1990 par A. Turq et M. Lenoir. Les niveaux paléolithiques sont constitués d'un épais ensemble d'Aurignacien récent. La surface fouillée est faible mais le matériel est bien conservé. La faune, étudiée par J.-C. Castel est constituée essentiellement de Cheval. Les différentes études archéologiques devraient être publiées dans des délais assez brefs

2. Une telle symbolique, quelque peu différente, est également présente durant les stades moyen et supérieur du Magdalénien occidental (Chauvière 2001 ; Pales 1969 ; Rousseau 1967 ; Rouzaud 1997).

\section{RÉSUMÉS}

Un récent examen, en vue de leur présentation muséographique, des collections de La Ferrassie (Savignac-de-Miremont, Dordogne) conservées au Musée national de Préhistoire des Eyzies, a permis de "re-découvrir », dans les niveaux aurignaciens, le retouchoir sur canine de grand carnivore ou « cousoir» qu'avaient publié L. Capitan et D. Peyrony dès 1912. A cette occasion, trois pièces semblables qu'accompagnent des dents jugales de cheval utilisées, ainsi que des retouchoirs sur diaphyses plus classiques, ont également été identifiés. A l'issue d'une analyse 
technique et comparative de ces pièces, replacées dans leur contexte historique et archéologique, est discutée l'utilisation de matériaux a priori différents pour des modes de fonctionnement apparemment similaires. Le statut des vestiges des grands carnivores est envisagé sur la base des données disponibles pour les gisements aurignaciens du sud-ouest de la France en particulier et pour le Paléolithique supérieur européen en général.

During a recent examination of the collections from La Ferrassie (Savignac-de-Miremont, Dordogne), conserved at the National Museum of Prehistory, we "re-discovered» in the Aurignacian levels, the retoucher on a large carnivore canine, or "cousoir ", published by L. Capitan and D. Peyrony in 1912. Three similar objects associated with used jugal horse teeth, as well as more typical retouchers on diaphyses, were also identified. Following a technological and comparative analysis of these objects, replaced in their historic and archaeological context, we discuss the use of materials that are a priori different for apparently similar functions. The status of large carnivore remains is considered based on available data from Aurignacian sites in southwest France in particular, and for the European Upper Paleolithic in general.

\section{INDEX}

Mots-clés : Aurignacien ancien, La Ferrassie, retouchoirs, Panthera spelaea, Ursus spelaeus, Equus caballus, Ongulés, canines, molaires, os, gestuelle technique

Keywords : Early Aurignacian, La Ferrassie, retouchers, Panthera spelaea, Ursus spelaeus, Equus caballus, Ungulates, canines, molars, bone, technical gestures

\section{AUTEURS}

\section{JEAN-CHRISTOPHE CASTEL}

Département d'archéozoologie - Muséum d'histoire naturelle - Route de Malagnou 1 CP 6434 1211 Genève 6 - Suisse. mel : jean-christophe.castel@mhn.ville-ge.ch

\section{FRANÇOIS-XAVIER CHAUVIÈRE}

Institut de préhistoire, Laténium, Parc et musée d'archéologie, Espace Paul Vouga, 2068

Hauterive, Suisse. mel : francois-xavier.chauviere@ne.ch et francois-xavier.chauviere@unine.ch

\section{STÉPHANE MADELAINE}

Stéphane Madelaine, Musée Nal de Préhistoire - 24620 Les Eyzies. - Université Bordeaux I - UMR 5808 - Institut de Préhistoire et de Géologie du Quaternaire, av. des Facultés - 33405 Talence cedex. mel : stephane.madelaine@culture.gouv.fr 